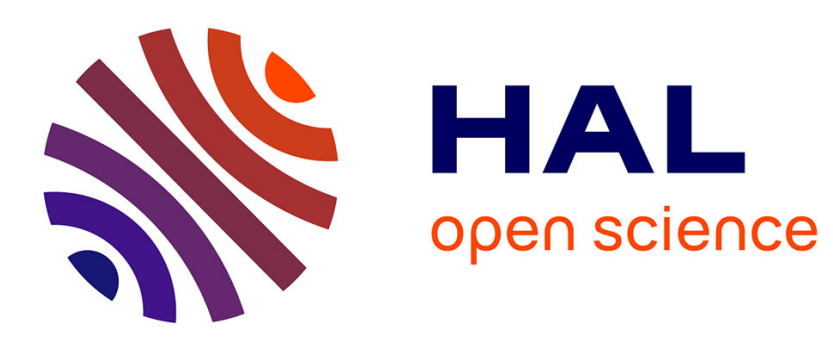

\title{
Existence of surface acoustic waves in one-dimensional piezoelectric phononic crystals of general anisotropy
}

\author{
A.N. Darinskii, A.L. Shuvalov
}

\section{To cite this version:}

A.N. Darinskii, A.L. Shuvalov. Existence of surface acoustic waves in one-dimensional piezoelectric phononic crystals of general anisotropy. Physical Review B: Condensed Matter and Materials Physics (1998-2015), 2019, 99 (17), 10.1103/PhysRevB.99.174305 . hal-02396050

\section{HAL Id: hal-02396050 \\ https://hal.science/hal-02396050}

Submitted on 14 Dec 2020

HAL is a multi-disciplinary open access archive for the deposit and dissemination of scientific research documents, whether they are published or not. The documents may come from teaching and research institutions in France or abroad, or from public or private research centers.
L'archive ouverte pluridisciplinaire HAL, est destinée au dépôt et à la diffusion de documents scientifiques de niveau recherche, publiés ou non, émanant des établissements d'enseignement et de recherche français ou étrangers, des laboratoires publics ou privés. 


\title{
Existence of surface acoustic waves in one-dimensional piezoelectric phononic crystals of general anisotropy
}

\author{
A. N. Darinskii ${ }^{1}$ and A. L. Shuvalov ${ }^{2}$ \\ ${ }^{1}$ Institute of Crystallography FSRC "Crystallography and Photonics” \\ Russian Academy of Sciences, Leninskii pr. 59, Moscow 119333, Russia \\ ${ }^{2}$ Université Bordeaux, CNRS, UMR 5295, Talence 33405, France
}

(Received 21 January 2019; revised manuscript received 15 April 2019; published 14 May 2019)

\begin{abstract}
The paper is concerned with the surface acoustic waves propagating in half-infinite one-dimensional piezoelectric phononic crystals of general anisotropy. The phononic crystal is formed of periodically repeated perfectly bonded layers, and its exterior boundary is one of the layer boundaries. The surface waves occurring in the so-called full stop bands are considered. It appears that the number of surface waves existing within a full stop band for a given layered structure is interrelated with their number in the same stop band for the phononic crystal different from the given one only due to the reversed ordering of layers within a period. A series of statements is proved on the maximum possible number of surface waves per full stop band for both these structures in total, i.e., embracing surface-wave occurrences in either one or the other structure. The analysis is performed for the electrically closed, electrically open, and electrically free types of boundary conditions on the mechanically free crystal surface, in which cases it admits a correspondingly different number of surface waves. A subsidiary instance of mechanically clamped surface is addressed as well. It is observed that the piezoelectric coupling can create new surface waves which disappear when piezoelectric coefficients turn to zero. Besides the general case of arbitrary anisotropy, we also consider two specific situations where the crystallographic symmetry allows the existence of sagittally polarized piezoactive surface waves and of shear horizontally polarized piezoactive surface waves. Numerical examples of surface-wave branches under various boundary conditions are provided.
\end{abstract}

DOI: 10.1103/PhysRevB.99.174305

\section{INTRODUCTION}

The piezoelectric effect is one of the fundamental physical properties of anisotropic media which exists in dielectric and semiconductor materials without the center of inversion (except those of 432 symmetry class) [1-3]. The coupling between mechanical and electric fields occurring in piezoelectric materials essentially influences various phenomena, underlies new physical effects, and paves the way to miscellaneous applications in different areas of technology [3]. The piezoelectric effect shows up in bulk and surface physical processes. In particular, it can markedly change the velocity and polarization of the bulk elastic modes propagating in piezoelectric solids [4,5]. A new channel of the phonon-current carriers' interaction comes about in piezoelectric semiconductors because of the electric field accompanying mechanical vibrations [6-9]. On the one hand, such interaction increases the phonon attenuation and decreases the current carrier mobility [10-12]. On the other hand, by applying a high dc electric field which makes electrons or holes move with the velocity exceeding the velocity of the elastic wave, one can use this interaction to amplify elastic waves and effectively generate coherent phonons; see, e.g., [8,13]. Moreover, the piezoelectric effect allows as well a direct generation of elastic waves by ac electric fields which is widely used in practice [14]. Besides these examples, the piezoelectric coupling can lead to the formation of polaritons of specific types [15-18].

Piezoelectricity driven modifications of the surface dynamics are also quite remarkable. Apart from the analogs of above-listed bulk phenomena, the piezoelectric effect gives rise to surface acoustic waves (SAWs) not existing in nonpiezoelectric solids. The SAWs generated by ac electric fields in piezoelectric substrates are intensively used in modern telecommunication systems [19]. The electric field of SAWs propagating in a piezoelectric semiconductor is known to affect the exciton dissociation in quantum wells [20]. Another series of effects occurs due to the fact that the surface vibrations induce the electric field not only inside piezoelectric materials but also in the exterior space. This electric field can couple waves in two piezoelectric solids separated by a vacuum gap. As a result, the gap can guide a special type of localized waves commonly referred to as gap waves [4], and the bulk waves/phonons can penetrate from one piezoelectric to another through the gap. Due to the same tunneling mechanism, an additional channel of heat exchange appears [21].

The general theory of SAWs on homogeneous piezoelectric substrates of arbitrary anisotropy, approached in Refs. [22,23], was developed in full in Refs. [24,25] and independently, by using a somewhat different approach, in Ref. [26]. It was proved there that two SAWs can exist on a piezoelectric substrate, whereas at most one SAW is allowed on a purely elastic substrate $[27,28]$. The piezoelectric effect was shown to also change the maximum permissible number of interfacial waves at solid-solid contacts [29,30].

In recent years much effort has been made for studying bulk and surface acoustic waves in both the purely elastic and the piezoelectric man-made periodic structures called the 
phononic crystals [31-34]. In particular, many studies were devoted to the wave propagation in one-dimensional (1D) phononic crystals, also termed superlattices, which consist of periodically arranged layers [35]. The most numerous results were obtained for the shear horizontally polarized SAWs considered under different settings in phononic crystals of various compositions [36-45]. The sagittally polarized twopartial and the fully coupled three-partial SAWs were also widely investigated [46-54]. The spectra of SAWs along with the bulk-wave reflection and transmission coefficients were studied as well for periodic solid-fluid structures $[55,56]$ and for solid-solid and solid-fluid Fibonacci superlattices [57-60]. Results of the numerical modeling of SAW propagation in two-dimensional piezoelectric phononic crystals may be found in [61-63].

In Ref. [64], we have studied the existence of SAWs in half-infinite 1D purely elastic phononic crystals. It was proved that, for any given wave number, at most three SAWs can exist in a full stop band (i.e., in the spectral zone where no bulk modes can exist), this number embracing the SAWs which occur in the given phononic crystal and those which occur in the related phononic crystal of the same set of layers but arranged in the reversed order within a period. Only one SAW per full stop band can exist in the special case of a phononic crystal whose layer arrangement in a period is symmetric with respect to its midplane.

The present paper is concerned with the existence problem of SAWs in half-infinite 1D piezoelectric phononic crystals of general anisotropy under different types of electrical conditions supplied at the crystal surface, namely, under the electrically closed, electrically open, and electrically free boundary conditions. The impact of piezoelectric effect will be shown to increase the maximum permissible number of SAWs pera stop band as compared to the purely elastic case. Our analysis is going to be largely based on the analytical properties of the surface impedance and related matrices. The concept of the surface impedance and admittance matrices was introduced in Ref. [65] and developed in Refs. [24,25,27,28,66,67] as a key tool of the theory of SAWs in homogeneous elastic and piezoelectric anisotropic substrates. These matrices also appear fruitful for constructing recursive schemes of numerically stable modeling of SAW propagation in multilayered media; see, e.g., [52] (there is a large number of later publications on this issue, but it is beyond the scope of our paper). Algebraic and analytical properties of the surface impedance and admittance defined for a 1D elastic phononic crystal were established and applied to the SAW existence problem in Ref. [64]. In the present paper, we will elaborate this formalism for $1 \mathrm{D}$ piezoelectric phononic crystals.

The paper is organized as follows. The background of our study is introduced in Sec. II, where we outline some basics of the theory of acoustoelectric waves in piezoelectrics and formulate the boundary conditions to be considered further in the paper. In Sec. III we examine the possible number of SAWs per full stop band in the phononic crystals of general anisotropy and of certain types of crystallographic symmetry subjected to various boundary conditions. Numerical examples illustrating the analytical conclusions are provided in Sec. V. Section VI summarizes the results obtained. In the Appendix some auxiliary properties of the surface admittance and impedance of a half-infinite piezoelectric phononic crystal are proved.

\section{ACOUSTOELECTRIC WAVES IN PERIODIC PIEZOELECTRIC MATERIALS}

Consider a piezoelectric nonconductive medium characterized by the density $\rho$, the elastic stiffness tensor at constant electric field $c_{i j k l}^{E}$, the piezoelectric tensor $e_{i j k}$, and the dielectric permittivity tensor at constant strain $\varepsilon_{i j}^{S}(i, j, k, l=$ $1,2,3)$. The velocity of sound being four to five orders smaller than that of light, the quasielectrostatic approximation is commonly a good approximation for the description of electric fields accompanying elastic waves in piezoelectric materials $[4,5]$. Within this framework, the propagation of time-harmonic acoustoelectric waves with a frequency $\omega$ is described by the equations

$$
\frac{\partial \sigma_{i j}}{\partial x_{j}}=-\rho \omega^{2} u_{i}, \quad \frac{\partial D_{i}}{\partial x_{i}}=0,
$$

complemented by the constitutive relations

$$
\sigma_{i j}=c_{i j k l}^{E} \frac{\partial u_{k}}{\partial x_{l}}+e_{k i j} \frac{\partial \varphi}{\partial x_{k}}, \quad D_{i}=e_{i j k} \frac{\partial u_{j}}{\partial x_{k}}-\varepsilon_{i j}^{S} \frac{\partial \varphi}{\partial x_{j}},
$$

where $\sigma_{i j}$ are the components of the mechanical stress tensor $\hat{\sigma}, u_{i}$ are the components of the mechanical displacement $\mathbf{u}(\mathbf{r}, t), x_{i}$ are the components of the radius vector $\mathbf{r}, D_{i}$ are the components of the electric displacement $\mathbf{D}(\mathbf{r}, t)$, and $\varphi(\mathbf{r}, t)$ is the electric potential.

Assume the given medium to be multilayered. Let $\mathbf{m}$ be the unit vector parallel to the plane of layer interfaces and $\mathbf{n}$ be the unit vector orthogonal to this plane. A plane wave traveling in this medium with the wave number $k$ is sought in the form

$$
\left(\begin{array}{l}
\mathbf{u}(\mathbf{r}, \mathbf{t}) \\
\varphi(\mathbf{r}, t)
\end{array}\right)=\left(\begin{array}{l}
\mathbf{a}(y) \\
\phi(y)
\end{array}\right) e^{i(k x-\omega t)},
$$

where $y=(\mathbf{n r})$ and $x=(\mathbf{m r})$. By combining Eqs. (1) and (2), the displacement and potential amplitudes $\mathbf{a}(y)$ and $\phi(y)$ can be incorporated together with the amplitudes of the normal components of the stress and of the electric displacement,

$$
\left(\begin{array}{c}
\hat{\boldsymbol{\sigma}}(\mathbf{r}, t) \mathbf{n} \\
\mathbf{D}(\mathbf{r}, t) \cdot \mathbf{n}
\end{array}\right)=-i k\left(\begin{array}{c}
\mathbf{l}(y) \\
d(y)
\end{array}\right) e^{i(k x-\omega t)}
$$

to obey a system of eight first-order ordinary differential equations depending on the $y$ coordinate only. This system can be written in either of two equivalent forms $[24,25]$,

$$
\frac{1}{i k} \frac{d \xi_{P}}{d y}=\hat{\mathbf{N}}_{P} \xi_{P}, \quad P=\Phi, F,
$$

where $\xi_{\Phi}(y)=(\mathbf{a}, \phi, \mathbf{l}, d)^{t}$ and $\xi_{F}(y)=(\mathbf{a}, d, \mathbf{l}, \phi)^{t}$ are the eight-component state vectors of amplitudes (the superscript ${ }^{t}$ means transposition) and

$$
\begin{aligned}
& \hat{\mathbf{N}}_{\Phi} \\
& =-\left(\begin{array}{cc}
(n n)^{-1}(n m) & (n n)^{-1} \\
(m n)(n n)^{-1}(n m)-(m m)+\rho(\omega / k)^{2} \hat{\mathbf{I}}^{\prime} & (m n)(n n)^{-1}
\end{array}\right)
\end{aligned}
$$


is the $8 \times 8$ matrix built from $4 \times 4$ matrices $(a b)$ whose elements are contractions of the three-component vectors $\mathbf{a}, \mathbf{b}=\mathbf{n}$ or $\mathbf{m}$ with the material tensors of the medium, namely, $(a b)_{I J}=a_{k} E_{k I J l} b_{l}, I, J=1, \ldots, 4$, where $E_{k I J l}=$ $c_{k I J l}^{E}, I, J=1,2,3, \quad E_{k 4 J l}=e_{k J l}, J=1,2,3, E_{k I 4 l}=e_{l I k}$, $I=1,2,3, E_{k 44 l}=-\varepsilon_{k l}^{S}$. The symbol $\hat{\mathbf{I}}^{\prime}$ denotes the $4 \times 4$ matrix with three unit elements $I_{i i}^{\prime}=1, i=1,2,3$, the other ones being zero. The material tensors are piecewise constant functions of $y$ in that their values vary from one layer to another. The matrix $\hat{\mathbf{N}}_{F}$ is obtained from the matrix $\hat{\mathbf{N}}_{\Phi}$ by permuting fourth and eighth rows and fourth and eighth columns. Using two complementary $\Phi$ and $F$ representations facilitates the analysis of SAWs on piezoelectric substrates subjected to various electrical boundary conditions. Where appropriate, we will apply a common index $P=\Phi, F$ to both above representations.

In what follows we consider a $1 \mathrm{D}$ phononic crystal consisting of periodically repeated sequences of homogeneous piezoelectric nonconductive layers with a perfect mechanical and electrical bonding along their mutually parallel interfaces. According to Eq. (5) for $\xi_{\Phi}$ and to its analog for $\xi_{F}$, the unitcell transfer matrix transmitting the wave field amplitudes $\xi_{P}(y)$ through a period of $n$ layers is

$$
\hat{\mathbf{M}}_{P}=\hat{\mathbf{M}}_{P, n} \hat{\mathbf{M}}_{P, n-1} \ldots \hat{\mathbf{M}}_{P, 1},
$$

where $\hat{\mathbf{M}}_{P, m}=\exp \left(i k h_{m} \hat{\mathbf{N}}_{P, m}\right)$ is the transfer matrix through the $m$ th layer with the coefficient matrix $\hat{\mathbf{N}}_{P, m}$ and thickness $h_{m}$.

In view of the symmetry relation

$$
\hat{\mathbf{N}}_{P}=\hat{\mathbf{T}} \hat{\mathbf{N}}_{P}^{t} \hat{\mathbf{T}}, \quad P=\Phi, F,
$$

where

$$
\hat{\mathbf{T}}=\left(\begin{array}{cc}
\hat{\mathbf{O}} & \hat{\mathbf{I}} \\
\hat{\mathbf{I}} & \hat{\mathbf{O}}
\end{array}\right)
$$

with $\hat{\mathbf{I}}$ and $\hat{\mathbf{O}}$ being the $4 \times 4$ unit and zero matrices, respectively, the matrix (7) obeys the relation

$$
\hat{\mathbf{M}}_{P}^{-1}=\hat{\mathbf{T}} \hat{\mathbf{M}}_{P}^{\dagger} \hat{\mathbf{T}},
$$

where the symbol ${ }^{\dagger}$ denotes the Hermitian transpose.

Basic concepts of the theory of wave propagation in periodic media rest on the eigenproblem for the matrix $\hat{\mathbf{M}}_{P}$,

$$
\hat{\mathbf{M}}_{P} \zeta_{P, \alpha}=\gamma_{\alpha} \zeta_{P, \alpha}, \quad \alpha=1, \ldots, 8
$$

The eigenvalues $\gamma_{\alpha}$ are the same for both $\Phi$ and $F$ representations and the eigenvectors $\zeta_{P, \alpha}$ are composed as follows:

$$
\begin{aligned}
& \zeta_{\Phi, \alpha}=\left(\begin{array}{c}
\mathbf{U}_{\Phi, \alpha} \\
\mathbf{V}_{\Phi, \alpha}
\end{array}\right), \quad \mathbf{U}_{\Phi, \alpha}=\left(\begin{array}{c}
\mathbf{A}_{\alpha} \\
\Phi_{\alpha}
\end{array}\right), \quad \mathbf{V}_{\Phi, \alpha}=\left(\begin{array}{c}
\mathbf{L}_{\alpha} \\
D_{\alpha}
\end{array}\right), \\
& \zeta_{F, \alpha}=\left(\begin{array}{c}
\mathbf{U}_{F, \alpha} \\
\mathbf{V}_{F, \alpha}
\end{array}\right), \quad \mathbf{U}_{F, \alpha}=\left(\begin{array}{c}
\mathbf{A}_{\alpha} \\
D_{\alpha}
\end{array}\right), \quad \mathbf{V}_{F, \alpha}=\left(\begin{array}{c}
\mathbf{L}_{\alpha} \\
\Phi_{\alpha}
\end{array}\right),
\end{aligned}
$$

where the components $\mathbf{A}_{\alpha}, \Phi_{\alpha}, \mathbf{L}_{\alpha}$, and $D_{\alpha}$ have the same physical meaning as $\mathbf{a}, \phi, \mathbf{l}$, and $d$, respectively.

The algebraic properties of the eigenvalues and eigenvectors of $\hat{\mathbf{M}}_{P}$ are similar to those of the transfer matrix for nonpiezoelectric periodic structures $[53,68]$, since they are based on identity (10) common for both cases. By (10), the eigenvalues $\gamma_{\alpha}$ of $\hat{\mathbf{M}}_{P}$ appear in pairs satisfying either

$$
\left|\gamma_{\alpha}\right|=\left|\gamma_{\alpha+4}\right|=1 \text {, }
$$

or

$$
\gamma_{\alpha}=\frac{1}{\gamma_{\alpha+4}^{*}} \text { for }\left|\gamma_{\alpha}\right| \neq 1
$$

where ${ }^{*}$ means complex conjugate and $\alpha$ is fixed among $1, \ldots, 4$. Given the value of $k$, the frequency intervals, where at least one pair of eigenvalues satisfies (13), are called pass bands, and the frequency intervals, where all eight eigenvalues obey (14), will be called full stop bands. The orthonormalization and completeness relations following from Eq. (10) for a common case of a diagonalizable $\hat{\mathbf{M}}_{P}$ specify within a full stop band in the form

$$
\begin{gathered}
\zeta_{P, \alpha}^{\dagger} \hat{\mathbf{T}} \zeta_{P, \beta}=\delta_{\alpha+4, \beta}, \alpha=1, \ldots 4, \beta=1, \ldots, 8, \\
\sum_{\alpha=1}^{4}\left(\zeta_{P, \alpha} \otimes \hat{\mathbf{T}} \zeta_{P, \alpha+4}^{*}+\zeta_{P, \alpha+4} \otimes \hat{\mathbf{T}} \zeta_{P, \alpha}^{*}\right)=\left(\begin{array}{cc}
\hat{\mathbf{I}} & \hat{\mathbf{O}} \\
\hat{\mathbf{O}} & \hat{\mathbf{I}}
\end{array}\right),
\end{gathered}
$$

where $\delta_{\alpha, \beta}$ is the Kronecker symbol and $\otimes$ denotes the dyadic product.

A wave field taken at some reference coordinate $y_{0}$ can always be presented as a linear superposition of the eigenvectors $\zeta_{P, \alpha}$, and then this wave field at arbitrary $y$ is a similar superposition of the partial modes $\xi_{P, \alpha}(y)$, each being generated by the eigenvector $\zeta_{P, \alpha}$. The so-defined $\alpha$ th partial mode taken at the edge of the $p$ th period of thickness $H$ is $\xi_{P, \alpha}\left(y_{0}+p H\right)=\gamma_{\alpha}^{p} \zeta_{P, \alpha}$, i.e., the mode corresponding to the eigenvalue $\left|\gamma_{\alpha}\right|=1$ retains a constant absolute value at all period edges, while the absolute value of the mode with $\left|\gamma_{\alpha}\right| \neq$ 1 increases or decreases with growing number of periods. By analogy with the case of a homogeneous medium, the former and the latter partial modes can be called bulk and inhomogeneous, respectively. Thus, at least one pair of partial modes in a pass band are bulk ones, and all eight partial modes in a full stop band are inhomogeneous, four increasing and four decaying ones.

It is noteworthy that for any $k \neq 0$ there always exists a frequency interval $0<\omega<\omega_{u}$ which is a full stop band. This can be proved by invoking the time average internal energy $W_{\alpha}$ associated with an $\alpha$ th partial mode and integrating it over the period in the static limit $\omega=0$. Using Eq. (A6) of the Appendix yields

$$
\begin{aligned}
\left\langle W_{\alpha}\right\rangle_{\omega=0} & =\left.\int_{y_{0}}^{y_{0}+H} W_{\alpha}(y)\right|_{\omega=0} d y \\
& =-\frac{i k}{8}\left(\left|\gamma_{\alpha}\right|^{2}-1\right)\left(\mathbf{U}_{F, \alpha}^{\dagger} \mathbf{V}_{F, \alpha}-\mathbf{V}_{F, \alpha}^{\dagger} \mathbf{U}_{F, \alpha}\right)_{y_{0}} .
\end{aligned}
$$

Since $W_{\alpha}$ is strictly positive for all $\omega$ and $k$, except that it vanishes at both $\omega$ and $k$ being zero, assuming $k \neq 0$ and appealing to Eq. (17) shows that $\left|\gamma_{\alpha}\right| \neq 1$ for any $\alpha$ at $\omega=0$ and hence by continuity at nonzero $\omega<\omega_{u}$, where $\omega_{u}$ is some positive upper bound. It should also be added that the value $\omega=0$ is not a stop band/pass band border and therefore it differs from all other band edges in that zero frequency does not necessitate a degeneracy among the eigenvalues $\gamma_{\alpha}$ of $\hat{\mathbf{M}}_{P}$ and it keeps finite the matrix $\hat{\boldsymbol{\Xi}}_{P}$ (see Sec. III A). 
(a)

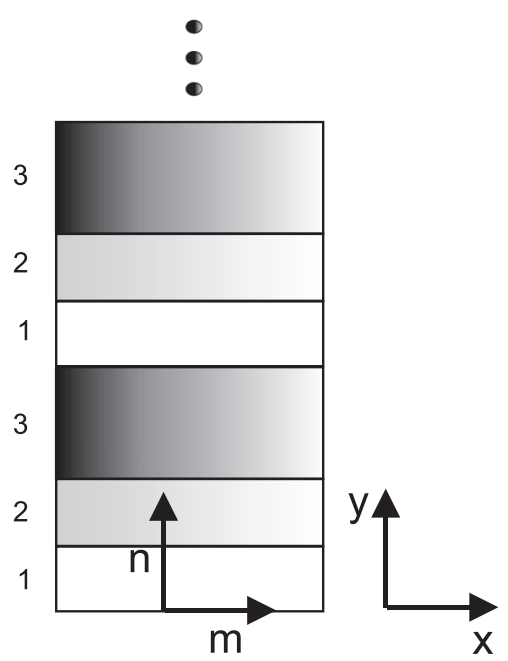

\section{$\mathrm{X}$}

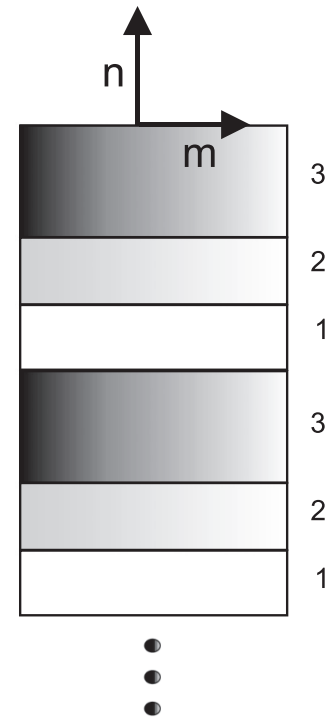

FIG. 1. Example of a "direct" half-infinite phononic crystal with the order of layers 1-2-3 (a) and the corresponding "reversed" halfinfinite phononic crystal with the order of layers is 3-2-1 (b).

Turning to the context of SAWs, we further specify the phononic crystal in hand as a periodic half space truncated at one of the layer surfaces, which is therefore natural to take as a period edge. In order that a SAW satisfies the mechanical and electrical boundary conditions at the halfspace surface, it should usually consist of four partial modes. Hence SAWs usually occur in the full stop bands. As we shall see, the existence of SAWs in a half-infinite phononic crystal $y>0$, characterized by an arbitrary given arrangement of layers within a period and referred to below as the "direct" phononic crystal [Fig. 1(a)], is interrelated to the existence of SAWs in the associated "reversed" phononic crystal, occupying the half space $y<0$ and consisting of the same layers as the direct phononic crystal but arranged within a period in the reversed order relatively to that in the direct phononic crystal [Fig. 1(b)]. The direct and reversed phononic crystals can be viewed as two halves of the infinite phononic crystal cut along a period edge. Given that the material tensors of both phononic crystals are defined with respect to the same coordinate system and the orientations of the vectors $\mathbf{m}$ and $\mathbf{n}$ are unchanged when passing from one structure to the other (see Fig. 1), the unit-cell matrix $\hat{\mathbf{M}}_{P}^{\prime}$ of the reversed phononic crystal is the inverse of unit-cell matrix $\hat{\mathbf{M}}_{P}$ of the direct phononic crystal,

$$
\hat{\mathbf{M}}_{P}^{\prime}=e^{-i k h_{1} \hat{\mathbf{N}}_{P, 1}} \ldots e^{-i k h_{n-1} \hat{\mathbf{N}}_{P, n-1}} e^{-i k h_{n} \hat{\mathbf{N}}_{P, n}}=\hat{\mathbf{M}}_{P}^{-1} .
$$

Thus the matrices $\hat{\mathbf{M}}_{P}^{\prime}$ and $\hat{\mathbf{M}}_{P}$ have the same eigenvectors $\zeta_{P, \alpha}$, while their eigenvalues are inverse of each other: $\gamma_{\alpha}^{\prime}=$ $1 / \gamma_{\alpha}, \alpha=1, \ldots, 8$, i.e., the direct and reversed phononic crystals share the same spectrum of pass bands and stop bands.

Note that the period of an infinite phononic crystal may be counted from any level on the periodicity axis, not necessarily coinciding with a layer interface. Therefore, the boundary surface of the periodic half space, which is a result of the cleavage of the infinite phononic crystal at its arbitrary level, can always be taken as a period edge. Correspondingly, all subsequent derivations equivally apply to a more general case than that depicted in Fig. 1, namely, the thickness of the external layer may be different from the thickness of the internal layers of the same material. The transfer matrix $\hat{\mathbf{M}}_{P}$ changes with changing the location of period edges and so do its eigenvectors. Hence, the characteristics of SAWs depend on whether the external layer has the same thickness as the similar internal layers or it is different. However, the eigenvalues of $\hat{\mathbf{M}}_{P}$ and hence the band spectrum are invariant to where the period edge is taken.

Considering in what follows the full stop bands, let us label the eigenvalues $\left|\gamma_{\alpha}\right|<1$ and $\left|\gamma_{\alpha}\right|>1$ by $\alpha=1,2,3,4$ and $\alpha=5,6,7,8$, respectively. Under this numbering, the former four eigenvalues generate the partial modes decaying at $y \rightarrow$ $\infty$ and hence constituting the SAWs in the direct phononic crystal, and the latter four eigenvalues generate the partial modes decaying at $y \rightarrow-\infty$ and constituting the SAWs in the reversed phononic crystal.

Sticking to the above-specified numbering of the partial modes, the SAW wave fields taken at the surface $y=0$ of the direct and reversed phononic crystals, respectively, can be written as

$$
\xi_{P}=\sum_{\alpha=1}^{4} b_{\alpha} \zeta_{P, \alpha}, \quad \xi_{P}^{\prime}=\sum_{\alpha=5}^{8} b_{\alpha}^{\prime} \zeta_{P, \alpha},
$$

where the coefficients $b_{\alpha}$ and $b_{\alpha}^{\prime}$ are to be found from the boundary conditions set at $y=0$. The main attention of this paper is paid to SAWs on the mechanically free surface with three different types of electrical conditions, while the occurrence of SAWs on the mechanically clamped surface is briefly discussed in Sec. III D.

Mechanically free electrically closed surface. This boundary condition implies the vanishing of the traction and electrical potential at $y=0$, that is,

$$
\sum_{\alpha=1}^{4} b_{\alpha} \mathbf{V}_{F, \alpha}=\mathbf{0}, \quad \sum_{\alpha=5}^{8} b_{\alpha}^{\prime} \mathbf{V}_{F, \alpha}=\mathbf{0}
$$

for the direct and reversed phononic crystals, respectively. In practice, the mechanically free electrically closed boundary condition is used to describe the traction-free surface which is coated with a thin metallic film such that it completely shields the electric field and at the same time does not produce any mechanical loading.

Mechanically free electrically open surface. In this case, the traction vanishes at $y=0$ along with the normal component of 
electric displacement, so that

$$
\sum_{\alpha=1}^{4} b_{\alpha} \mathbf{V}_{\Phi, \alpha}=\mathbf{0}, \quad \sum_{\alpha=5}^{8} b_{\alpha}^{\prime} \mathbf{V}_{\Phi, \alpha}=\mathbf{0}
$$

for the direct and reversed phononic crystals, respectively. This is a somewhat model case which is, however, of clear interest for several reasons. First, as it will be seen in Sec. III, the information regarding the existence of SAWs on the mechanically free electrically open surface is required for analyzing the existence of SAWs in the common case of a crystalvacuum interface. Second, this condition can be viewed as the limiting case of the boundary condition on a crystal-vacuum interface when the relative dielectric permittivity of the crystal is far greater than unity. Third, bearing in mind that the piezomagnetic effect is described by a third-order tensor with the same symmetry in indices as the piezoelectric tensor [1], we note that all considerations to be developed for the case of a piezoelectric phononic crystal with an electrically open surface can be carried over to the case of a piezomagnetic phononic crystal with a thin superconductive coating merely via interpreting $D_{n, \alpha}$ as the normal component of magnetic induction.

Mechanically and electrically free surface (crystal-vacuum interface). This setting describes the most common type of electrical contact which maintains continuity of the corresponding electric parameters. It implies that the acoustoelectric wave (3) taken in the direct phononic crystal $y>0$ is accompanied in the vacuum half space $y<0$ by the wave of electric potential $\varphi^{(v)}(\mathbf{r}, t)$ and displacement $\mathbf{D}^{(v)}(\mathbf{r}, t)$ such that

$$
\varphi^{(v)}=\Phi^{(v)} e^{k y+i(k x-\omega t)}, \quad \mathbf{D}^{(v)} \cdot \mathbf{n}=-\varepsilon_{0} k \varphi^{(v)},
$$

where $\varepsilon_{0}$ is the vacuum permittivity and $k>0$ is assumed. Thus the vanishing of traction and the continuity of potential and of normal component of electric displacement yield

$$
\sum_{\alpha=1}^{4} b_{\alpha} \mathbf{L}_{\alpha}=\mathbf{0}, \quad \sum_{\alpha=1}^{4} b_{\alpha} \Phi_{\alpha}=\Phi^{(v)}, \quad \sum_{\alpha=1}^{4} b_{\alpha} D_{\alpha}=-i \varepsilon_{0} \Phi^{(v)} .
$$

In the case of acoustoelectric wave in the reversed phononic crystal $y<0$, the accompanying quasielectrostatic wave in the vacuum half space $y>0$ is described by

$$
\varphi^{(v) \prime}(\mathbf{r}, t)=\Phi^{(v) \prime} e^{-k y+i(k x-\omega t)}, \quad \mathbf{D}^{(v) \prime} \cdot \mathbf{n}=\varepsilon_{0} k \varphi^{(v) \prime},
$$

hence the boundary condition at $y=0$ requires

$$
\sum_{\alpha=5}^{8} b_{\alpha}^{\prime} \mathbf{L}_{\alpha}=\mathbf{0}, \quad \sum_{\alpha=5}^{8} b_{\alpha}^{\prime} \Phi_{\alpha}=\Phi^{(v) \prime}, \quad \sum_{\alpha=5}^{8} b_{\alpha}^{\prime} D_{\alpha}=i \varepsilon_{0} \Phi^{(v) \prime} .
$$

Note that the boundary condition (23) or (25) applies as well to the case of a dielectric adjoined to the crystal without a mechanical contact. The only modification, which is due on adopting this case, is replacement of $\varepsilon_{0}$ with the relative permittivity $\varepsilon$ of the dielectric medium. Note also that one way to change the effective permittivity of the exterior from $\infty$ to $\varepsilon_{0}$ (i.e., to pass from the electrically closed to electrically free boundary conditions) is to adjust, without a mechanical contact, a conducting layer with an outer face at some distance $h$ from the crystal surface $y=0$. Given an acoustoelectric wave traveling with a wave number $k$ in the crystal, the effective dielectric permittivity of the exterior will be $\varepsilon=\varepsilon_{0} \operatorname{coth}(k h)$.

\section{EXISTENCE OF SURFACE WAVES}

\section{A. Electrically closed and open surfaces}

In accordance with Eqs. (20) and (21) an immediate form of the dispersion equations for SAW in the direct and the reversed phononic crystal is, respectively,

$$
\operatorname{det}\left(\hat{\mathbf{V}}_{P}\right)=0 \text { and } \operatorname{det}\left(\hat{\mathbf{V}}_{P}^{\prime}\right)=0,
$$

where the symbols $\hat{\mathbf{V}}_{P}$ and $\hat{\mathbf{V}}_{P}^{\prime}$ stand for the $4 \times 4$ matrices whose columns are the vectors $\mathbf{V}_{P, \alpha}, \alpha=1,2,3,4$, and $\mathbf{V}_{P, \alpha}$, $\alpha=5,6,7,8$, respectively. However, Eqs. (26) as well as certain other equivalent forms of the dispersion equations cited in the end of Sec. III A are not actually suitable for the analysis of the SAW existence.

Our approach will proceed from the dispersion equations, each of which embraces the occurrences of the SAWs in the direct and reversed phononic crystals simultaneously. For formulating and analyzing such equations, we introduce the matrix

$$
\begin{aligned}
\hat{\boldsymbol{\Xi}}_{P} & \equiv\left(\begin{array}{ll}
\hat{\mathbf{\Xi}}_{P, 11} & \hat{\mathbf{\Xi}}_{P, 12} \\
\hat{\mathbf{\Xi}}_{P, 21} & \hat{\mathbf{\Xi}}_{P, 22}
\end{array}\right) \\
& =i \sum_{\alpha=1}^{4}\left[\zeta_{P, \alpha} \otimes \hat{\mathbf{T}} \zeta_{P, \alpha+4}^{*}-\zeta_{P, \alpha+4} \otimes \hat{\mathbf{T}} \zeta_{P, \alpha}^{*}\right],
\end{aligned}
$$

which is defined within the full stop bands via the eigenvectors of $\hat{\mathbf{M}}_{P}$ normalized according to Eq. (15). The key element is its off-diagonal block $4 \times 4$ block $\hat{\mathbf{\Xi}}_{P, 21}$. By its construction as defined in (27), $\hat{\boldsymbol{\Xi}}_{P, 21}$ is a Hermitian matrix. Combining (27) with the completeness relation (16) yields

$$
\hat{\Xi}_{P, 21}=2 i \sum_{\alpha=1}^{4} \mathbf{V}_{P, \alpha} \otimes \mathbf{V}_{P, \alpha+4}^{*}=2 i \hat{\mathbf{V}}_{P} \hat{\mathbf{V}}_{P}^{\prime \dagger}
$$

and hence

$$
\operatorname{det}\left(\hat{\boldsymbol{\Xi}}_{P, 21}\right)=16 \operatorname{det}\left(\hat{\mathbf{V}}_{P}\right) \operatorname{det}\left(\hat{\mathbf{V}}_{P}^{* *}\right) .
$$

Therefore

$$
\operatorname{det}\left(\hat{\boldsymbol{\Xi}}_{F, 21}\right)=0
$$

and

$$
\operatorname{det}\left(\hat{\boldsymbol{\Xi}}_{\Phi, 21}\right)=0
$$

are the dispersion equations for SAWs on the mechanically free electrically closed and open surfaces, respectively, each of these equations embracing the SAW occurrences in the direct and the reversed phononic crystals.

In the following derivations, we will also use the upper offdiagonal block $\hat{\boldsymbol{\Xi}}_{P, 12}$ of (27). It is also a Hermitian matrix and its definition by Eq. (27) admits an equivalent representation

$$
\hat{\Xi}_{P, 12}=2 i \sum_{\alpha=1}^{4} \mathbf{U}_{P, \alpha} \otimes \mathbf{U}_{P, \alpha+4}^{*}=2 i \hat{\mathbf{U}}_{P} \hat{\mathbf{U}}_{P}^{\prime \dagger}
$$


where $\hat{\mathbf{U}}_{P}$ and $\hat{\mathbf{U}}_{P}^{\prime}$ denote the $4 \times 4$ matrices whose columns are the vectors $\mathbf{U}_{P, \alpha}, \alpha=1,2,3,4$, and $\mathbf{U}_{P, \alpha}, \alpha=5,6,7,8$, respectively.

Additional insight into the properties of the matrices $\hat{\boldsymbol{\Xi}}_{P, 21}$ and $\hat{\mathbf{\Xi}}_{P, 12}$ can be gained via the matrices of surface admittance $\hat{\mathbf{Y}}_{P}, \hat{\mathbf{Y}}_{P}^{\prime}$ and impedance $\hat{\mathbf{Z}}_{P}=\hat{\mathbf{Y}}_{P}^{-1}, \hat{\mathbf{Z}}_{P}^{\prime}=\hat{\mathbf{Y}}_{P}^{\prime-1}$ of the direct and reversed phononic crystals, respectively. We introduce the admittances by the definition

$$
\begin{aligned}
& \mathbf{U}_{P, \alpha}=i \hat{\mathbf{Y}}_{P} \mathbf{V}_{P, \alpha}, \quad \alpha=1, \ldots, 4, \\
& \mathbf{U}_{P, \alpha}=-i \hat{\mathbf{Y}}_{P}^{\prime} \mathbf{V}_{P, \alpha}, \quad \alpha=5, \ldots, 8,
\end{aligned}
$$

and the impedances follow as the inverse of (33). Combining this definition with the orthogonality property (15) shows that the admittance and impedances in the full stop bands are Hermitian matrices. Moreover, by manipulating (33) together with (15) and comparing the result with (28) and (32), or else by observing from Eqs. (27) and (15) that

$$
\begin{aligned}
& \hat{\mathbf{\Xi}}_{P} \zeta_{P, \alpha}=i \zeta_{P, \alpha}, \quad \alpha=1, \ldots, 4, \\
& \hat{\mathbf{\Xi}}_{P} \zeta_{P, \alpha}=-i \zeta_{P, \alpha}, \quad \alpha=5, \ldots, 8,
\end{aligned}
$$

and then using these relations to express the admittances and impedances via the blocks of $\hat{\boldsymbol{\Xi}}_{P}$, one can verify that

$$
\hat{\mathbf{\Xi}}_{P, 21}=2\left(\hat{\mathbf{Y}}_{P}+\hat{\mathbf{Y}}_{P}^{\prime}\right)^{-1}, \quad \hat{\mathbf{\Xi}}_{P, 12}=-2\left(\hat{\mathbf{Z}}_{P}+\hat{\mathbf{Z}}_{P}^{\prime}\right)^{-1} .
$$

The above link allows us to take advantage of the signdefiniteness property of the frequency derivative of the admittance and impedance matrices, which is established in the Appendix [see Eqs. (A3) and (A4)]. It reads that

$\frac{\partial \hat{\mathbf{Y}}_{P}}{\partial \omega}$ and $\frac{\partial \hat{\mathbf{Y}}_{P}^{\prime}}{\partial \omega}$ are positive definite matrices in full stop bands.

$\frac{\partial \hat{\mathbf{Z}}_{P}}{\partial \omega}$ and $\frac{\partial \hat{\mathbf{Z}}_{P}^{\prime}}{\partial \omega}$ are negative definite matrices in full stop bands

Hence, by virtue of Eq. (35),

$$
\begin{aligned}
& \frac{\partial \hat{\boldsymbol{\Xi}}_{P, 21}}{\partial \omega} \text { and } \frac{\partial \hat{\boldsymbol{\Xi}}_{P, 12}}{\partial \omega} \\
& \text { are negative definite matrices in full stop bands. }
\end{aligned}
$$

Further to this point, denote the real eigenvalues and unitnormalized eigenvectors of $\hat{\mathbf{\Xi}}_{P, 21}$ by $\tau_{P, \alpha}$ and $\mathbf{e}_{P, \alpha}$, respectively. The spectral decomposition $\hat{\mathbf{\Xi}}_{P, 21}=\sum_{\alpha=1}^{4} \tau_{P, \alpha} \mathbf{e}_{P, \alpha} \otimes$ $\mathbf{e}_{P, \alpha}^{*}$ and the orthogonalities $\mathbf{e}_{P, \alpha}^{\dagger} \mathbf{e}_{P, \beta}=\delta_{\alpha \beta},\left(\partial \mathbf{e}_{P, \alpha}^{\dagger} / \partial \omega\right) \mathbf{e}_{P, \alpha}=$ 0 validate the equality $\mathbf{e}_{P, \alpha}^{\dagger}\left(\partial \hat{\mathbf{\Xi}}_{P, 21} / \partial \omega\right) \mathbf{e}_{P, \alpha}=\partial \tau_{P, \alpha} / \partial \omega$. The Hermitian quadratic form on the left-hand side is negative in the full stop bands due to Eq. (38) $)_{1}$. Hence

$$
\frac{\partial \tau_{P, \alpha}}{\partial \omega}<0, \alpha=1,2,3,4, \text { in the full stop bands. }
$$

The derivatives of the eigenvalues of $\hat{\boldsymbol{\Xi}}_{P, 12}$ possess analogous properties.

All elements of the matrix $\hat{\boldsymbol{\Xi}}_{P}$ (27) are finite everywhere inside the full stop bands, including possible points of degeneracy of the eigenvalues of $\hat{\mathbf{M}}_{P}$ at which the finiteness of $\hat{\boldsymbol{\Xi}}_{P}$ is not obvious but still can be shown to hold true (see a similar derivation for the pure elastic case in Ref. [64]). Therefore the eigenvalues of the matrices $\hat{\mathbf{\Xi}}_{P, 21}$ and $\hat{\mathbf{\Xi}}_{P, 12}$ are finite and so they are monotonically decreasing functions of frequency in the full stop bands.

Coming back now to the dispersion equations (30) and (31), it follows from the monotonicity of $\tau_{P, \alpha}(\omega)$ and Eq. (39) that each of the $\tau_{P, \alpha}$ vanishes not more than once within a full stop band. Therefore, each of equations (30) and (31) can have at most four roots per full stop band. It is essential to add that a possible simultaneous vanishing of $\operatorname{both} \operatorname{det}\left(\hat{\mathbf{V}}_{P}\right)$ and $\operatorname{det}\left(\hat{\mathbf{V}}_{P}^{\prime}\right)$ at the same frequency, i.e., coexistence of the SAW in the direct phononic crystal with the SAW in the reversed one, cannot be due to vanishing of only one eigenvalue $\tau_{P, \alpha}$. Indeed, in view of the fact that the frequency derivatives of $\operatorname{det}\left(\hat{\mathbf{V}}_{P}\right)$ and $\operatorname{det}\left(\hat{\mathbf{V}}_{P}^{\prime}\right)$ are finite, the occurrence of a common zero of $\operatorname{det}\left(\hat{\mathbf{V}}_{P}\right)$ and $\operatorname{det}\left(\hat{\mathbf{V}}_{P}^{\prime}\right)$ at some frequency entails a zero-frequency derivative of $\operatorname{det}\left(\hat{\boldsymbol{\Xi}}_{P, 21}\right)=\prod_{i=1}^{4} \tau_{P, i}$ at this frequency. By virtue of Eq. (39), $\partial \tau_{P, \alpha} / \partial \omega \neq 0$. Hence, at least two eigenvalues $\tau_{P, \alpha}$ must turn zero. Thus any zero of each eigenvalue $\tau_{P, \alpha}$ corresponds to one and only one SAW, which propagates in either the direct or the reversed phononic crystal.

On these grounds, we conclude that, given the value of $k$,

at most four SAWs within a full stop band can occur in total in the direct and reversed phononic crystal with mechanically free electrically closed surface;

at most four SAWs within an upper full stop band can occur in total in the direct and reversed phononic crystals with mechanically free electrically open surface.

Statements (40) and (41) proceed from a possibility of $\hat{\boldsymbol{\Xi}}_{P, 21}$ to be positive-definite at the lower edge of a given stop band and in this the four eigenvalues of $\hat{\boldsymbol{\Xi}}_{P, 21}$ are positive near the lower edge. It can always be the case in principle, except for the matrix $\hat{\boldsymbol{\Xi}}_{\Phi, 21}$ at the edge $\omega=0$ of the lowest stop band $0<\omega<\omega_{u}$. The latter is the reason for a restrictive reservation of (41) to any upper full stop band, which implies any full stop band except the lowest one. In fact the case of lowest stop band $0<\omega<\omega_{u}$ requires a special discussion due to the additional sign-definiteness properties of $\hat{\boldsymbol{\Xi}}_{P, 21}$ at $\omega=0$.

By virtue of Eqs. (A7) and (A8) of the Appendix,

$\hat{\mathbf{Y}}_{F}, \quad \hat{\mathbf{Y}}_{F}^{\prime}, \quad \hat{\mathbf{Z}}_{F}$,

and $\hat{\mathbf{Z}}_{F}^{\prime}$ are positive-definite matrices at $\omega=0$.

Therefore, in view of Eq. (35),

$$
\begin{aligned}
& \hat{\mathbf{\Xi}}_{F, 21} \text { is a positive-definite matrix at } \omega=0, \\
& \hat{\boldsymbol{\Xi}}_{F, 12} \text { is a negative-definite matrix at } \omega=0 .
\end{aligned}
$$

According to Eqs. (30) and (43) 1 , the case of lowest stop band in a phononic crystal with electrically closed surface precisely fits statement (40).

The matrices $\hat{\mathbf{Y}}_{\Phi}$ and $\hat{\mathbf{Y}}_{\Phi}^{\prime}$ and, as a result, $\hat{\boldsymbol{\Xi}}_{\Phi, 21}$ are not sign-definite at $\omega=0$ (see the Appendix). However, looking at Eqs. (12), (28), and (32) one can notice that the upper diagonal $3 \times 3$ block of the matrix $\hat{\boldsymbol{\Xi}}_{\Phi, 21}$ coincides with the similar block of the matrix $\hat{\boldsymbol{\Xi}}_{F, 21}$ and hence is 
positive-definite at $\omega=0$, due to Eq. (43) $)_{1}$. Besides, the diagonal 44th component of $\hat{\mathbf{\Xi}}_{\Phi, 21}$ is equal to the similar component of $\hat{\boldsymbol{\Xi}}_{F, 12}$ and, hence, is negative at $\omega=0$, due to Eq. $(43)_{2}$. From these two features of the matrix $\hat{\boldsymbol{\Xi}}_{\Phi, 21}$, it follows that three of its eigenvalues are positive and one is negative at $\omega=0$. Thus statement (41) can be supplemented by the following conclusion:

at most three SAWs within the lowest stop band can occur in total in the direct and reversed phononic crystals with mechanically free electrically open surface.

The case of the lowest stop band admits another particular result, namely, the existence criterion of SAWs in a structure with electrically closed surface. On the one hand, this criterion is based on Eq. (43) ${ }_{1}$ which implies that all eigenvalues of $\hat{\boldsymbol{\Xi}}_{F, 21}$ are positive at $\omega=0$. On the other hand, it exploits the behavior of $\hat{\boldsymbol{\Xi}}_{F, 21}$ in the vicinity of the stop band upper edge $\omega_{u}$. To this end, we note that the matrix $\hat{\boldsymbol{\Xi}}_{P}$ usually diverges at the stop band/pass band edges, where two eigenvalues $\gamma_{\alpha}$ and $\gamma_{\alpha+4}=1 / \gamma_{\alpha}^{*}$ of the unit-cell matrix $\hat{\mathbf{M}}_{P}$ degenerate into one eigenvalue $\gamma_{d}$ with $\left|\gamma_{d}\right|=1$ and two corresponding eigenvectors $\zeta_{P, \alpha}$ and $\zeta_{P, \alpha+4}$ merge into one eigenvector $\zeta_{P, d}=$ $\left(\mathbf{U}_{P, d} \mathbf{V}_{P, d}\right)^{t}$. This eigenvector generates a bulk partial mode with energy flow parallel to the layer interfaces which may be called a limiting wave, by analogy with the case of a homogeneous half space $[24,25,69]$. Using the perturbation theory similarly as it has been done with respect to the purely elastic structures in Ref. [64], it can be specified that at least one eigenvalue of $\hat{\boldsymbol{\Xi}}_{F, 21}$ tends to minus infinity as $\omega \rightarrow$ $\omega_{u}-0$, unless in the exceptional case when the limiting wave satisfies the boundary condition $\mathbf{V}_{F, d}=\mathbf{0}$ at the mechanically free electrically closed surface. On these grounds, we can conclude that

\begin{abstract}
at least one SAW exists within the lowest stop band in either the direct and the reversed phononic crystal with mechanically free electrically closed surface, unless the limiting wave at $\omega_{u}$ satisfies the boundary condition at this surface.
\end{abstract}

The limiting wave usually does not satisfy the boundary condition, hence one SAW in one or the other phononic crystal usually does exist. Note that a similar criterion cannot be extended to SAWs possibly occurring in the lowest stop band under the electrically open condition or to SAWs in the upper stopbands under any boundary conditions, because none of these cases ensure that all four eigenvalues of the matrix appearing in the corresponding dispersion equation are positive at stop band lower edge.

We note that the dispersion equations equivalent to Eqs. (30) and (31) can be formulated in terms of the impedances in the form $\operatorname{det}\left(\hat{\mathbf{Z}}_{P}\right)=0$ and $\operatorname{det}\left(\hat{\mathbf{Z}}_{P}^{\prime}\right)=0$ for the direct and reversed phononic crystals with mechanically free electrically closed or open surface. However, this formulation is not suitable for considerations of the SAW existence. The point is that the impedance eigenvalues may have poles, which occur within the full stop bands at the frequencies of SAWs supported by the mechanically clamped surface (see Sec. III D). Hence, the sign-definiteness properties (37) do not guarantee that an eigenvalue of $\hat{\mathbf{Z}}_{P}$ or $\hat{\mathbf{Z}}_{P}^{\prime}$ cannot have more than one zero within a full stop band.

In view of definitions (12) and (33), another equivalent form of the dispersion equations for piezoactive SAWs in a given phononic crystal with mechanically free electrically closed or open surface is $Y_{\Phi, 44}=0$ and $Y_{F, 44}=0$, respectively, where $Y_{P, 44}$ is the 44th component of the admittance $\hat{\mathbf{Y}}_{P}$. Similarly to the previous example, these equations on their own do not reveal a possible number of SAWs. At the same time, their use allows us to illuminate a sequence of the SAW frequencies for the electrically closed and open surfaces. According to the identity $Y_{\Phi, 44}=-1 / Y_{F, 44}$ which also follows from Eqs. (12) and (33), the zeros and poles of $Y_{\Phi, 44}$ in a full stop band are associated with SAWs on the mechanically free electrically closed and electrically open surface, respectively, while the inverse correspondence holds for $Y_{F, 44}$. Thus the difference between the neighboring zeros and poles of any of these functions $Y_{P, 44}$ is the measure of piezoelectric coupling in the sense of [22]. By virtue of Eq. (36), $\partial Y_{P, 44} / \partial \omega>0$, so the zeros and poles of $Y_{P, 44}$ follow one after another in frequency. Hence any two frequencies of SAWs, occurring at some fixed $k$ in a given phononic crystal with mechanically free electrically closed surface, envelope one frequency of SAW, occurring at this $k$ in the same crystal with mechanically free electrically open surface, and vice versa. These properties of the component $Y_{P, 44}$ of $4 \times 4$ admittance of a periodic half space are similar to the properties of eigenvalues of $2 \times 2$ blocks of $8 \times 8$ admittance of transversely inhomogeneous piezoelectric plates; see [70].

\section{B. Electrically free boundary condition}

A handy form of the dispersion equation for piezoactive SAWs on a crystal-vacuum interface can be obtained by introducing the mechanically free boundary condition $(23)_{1}$ into the definition of, say, $F$ admittance following from (33), which provides the relation $\sum_{\alpha=1}^{4} b_{\alpha} D_{\alpha}=$ $i Y_{F, 44}\left(\sum_{\alpha=1}^{4} b_{\alpha} \Phi_{\alpha}\right)$. Inserting here the electrical part of the boundary condition (23) and then repeating the same procedure using the boundary condition (25) leads to the sought dispersion equations for the direct and reversed phononic crystals, respectively, in the form

$$
Y_{F, 44}=-\varepsilon_{0}, Y_{F, 44}^{\prime}=-\varepsilon_{0} .
$$

The curves $Y_{F, 44}$ and $Y_{F, 44}^{\prime}$ within a full stop band have a positive frequency derivative [see Eq. (36)], which makes the dispersion equation in the form (46) convenient for graphical analysis. 


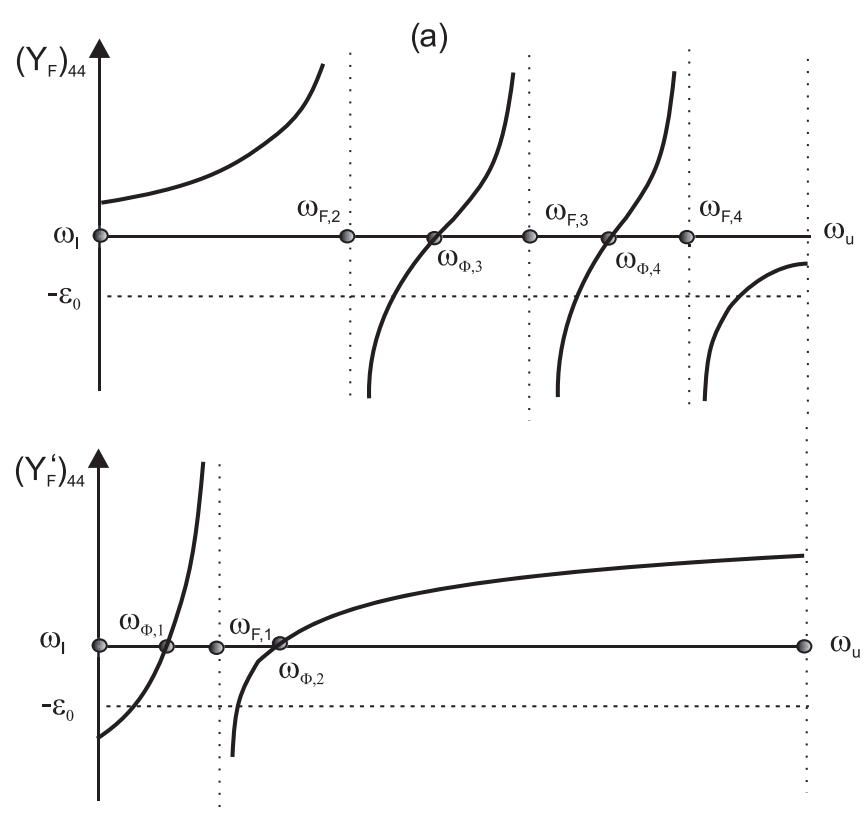

(b)
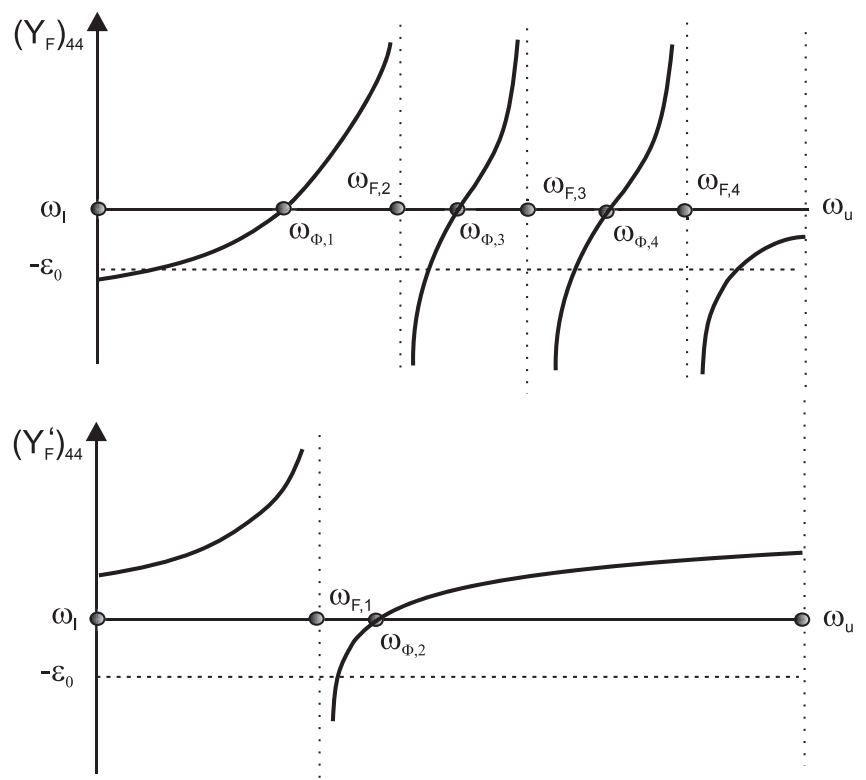

FIG. 2. Possible frequency dependencies of $Y_{F, 44}$ and $Y_{F, 44}^{\prime}$ in an upper full stop band, which ensure the existence of five SAWs within this stop band in total in the direct and reversed phononic crystals bounded by vacuum.

An example is provided in Fig. 2, where two possible patterns of the frequency dependence of $Y_{F, 44}$ and $Y_{F, 44}^{\prime}$ at a fixed $k$ are shown such that they admit five intersections of the curves $Y_{F, 44}$ and $Y_{F, 44}^{\prime}$ with the straight line $-\varepsilon_{0}$, i.e., five roots of Eqs. (46) $)_{1}$ and (46) $)_{2}$ in total, within an upper stop band $\omega_{l}<\omega<\omega_{u}\left(\omega_{l} \neq 0\right)$. Aiming to establish a maximum possible number of SAWs per stop band in the direct and reversed phononic crystals, we note from this graphical perspective that the prerequisite for a maximum number of roots of Eqs. (46) is a maximum number of zeros and poles of the functions $Y_{F, 44}$ and $Y_{F, 44}^{\prime}$. As mentioned in the end of Sec. III A, the zeros and poles of $Y_{F, 44}$ and of
$Y_{F, 44}^{\prime}$ indicate the SAW solutions on the mechanically free electrically open surface and electrically closed surface, respectively. In consequence, according to statements (40) and (41), the maximum permissible number of zeros and poles of the functions $Y_{F, 44}$ and $Y_{F, 44}^{\prime}$ per upper full stop band in total is 4. This exactly corresponds to the case displayed in Fig. 2, where both plotted patterns assume four zeros and four poles split one way or another between the $Y_{F, 44}$ and $Y_{F, 44}^{\prime}$ curves. Hence we may suppose that the maximum number of SAWs for a fixed $k$ and frequency varying within an upper full stop band is 5 .

Yet, in order to make sure that the number of SAWs cannot be more than 5, we need to refute one putative option which may seem plausible in the course of inspecting various modifications of Fig. 2. One may notice that six intersections of the $Y_{F, 44}$ and $Y_{F, 44}^{\prime}$ curves with a straight line $-\varepsilon_{0}$ could occur if the functions $Y_{F, 44}$ and $Y_{F, 44}^{\prime}$ having the maximum total number of four zeros and four poles within an upper full stop band would both be negative near the lower stop band edge $\omega_{l}$. However, this is impossible. Indeed, the assumed occurrence of, e.g., four poles of $Y_{F, 44}$ and $Y_{F, 44}^{\prime}$ in total implies that in the immediate vicinity of $\omega_{l}$ the matrix $\hat{\mathbf{\Xi}}_{F, 21}$ and, hence, the matrix $\hat{\boldsymbol{\Xi}}_{F, 21}^{-1}$ are positive-definite. In view of (35), in this instance $Y_{F, 44}+Y_{F, 44}^{\prime}$ must be positive, so $Y_{F, 44}$ and $Y_{F, 44}^{\prime}$ cannot both be negative. Thus five SAWs observed in Fig. 2 is really their maximum possible number within any upper stop band, and we are in the position to formulate the following statement: Given the value of $k$,

at most five SAWs within an upper full stop band can occur in total in the direct and reversed phononic crystals bounded by vacuum.

Passing to the case of lowest stop band $0<\omega<\omega_{u}$, we note that, in view of Eq. (44), the curves $Y_{F, 44}$ and $Y_{F, 44}^{\prime}$ in total can have at most three zeros within the lowest stop band. In addition, both $Y_{F, 44}$ and $Y_{F, 44}^{\prime}$ are positive at $\omega=0$ by Eq. (42). These properties are incorporated in Fig. 3 which visualizes the maximum possible number of four SAWs at a fixed $k$ in the lowest stop band. All other admissible configurations of the $Y_{F, 44}$ and $Y_{F, 44}^{\prime}$ curves yield the same conclusion. Hence we can formulate the following statement:

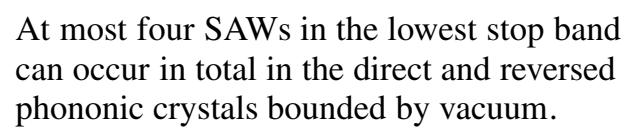

Interestingly, one SAW on the phononic crystal-vacuum interface of the direct and/or of the reversed phononic crystals can come about within an upper full stop band even when no SAWs exist for both the mechanically free electrically closed and electrically open boundary conditions. Such a situation can occur provided that $Y_{F, 44}<0$ and/or $Y_{F, 44}^{\prime}<0$ at $\omega=\omega_{l}$. It is impossible in the lowest stop band, since $Y_{F, 44}>0$ and $Y_{F, 44}^{\prime}>0$ at $\omega=0$.

\section{Effect of crystallographic symmetry}

No assumptions of any crystallographic symmetry of the layer materials have been used in the foregoing derivations. Let us now discuss two particular cases where the 

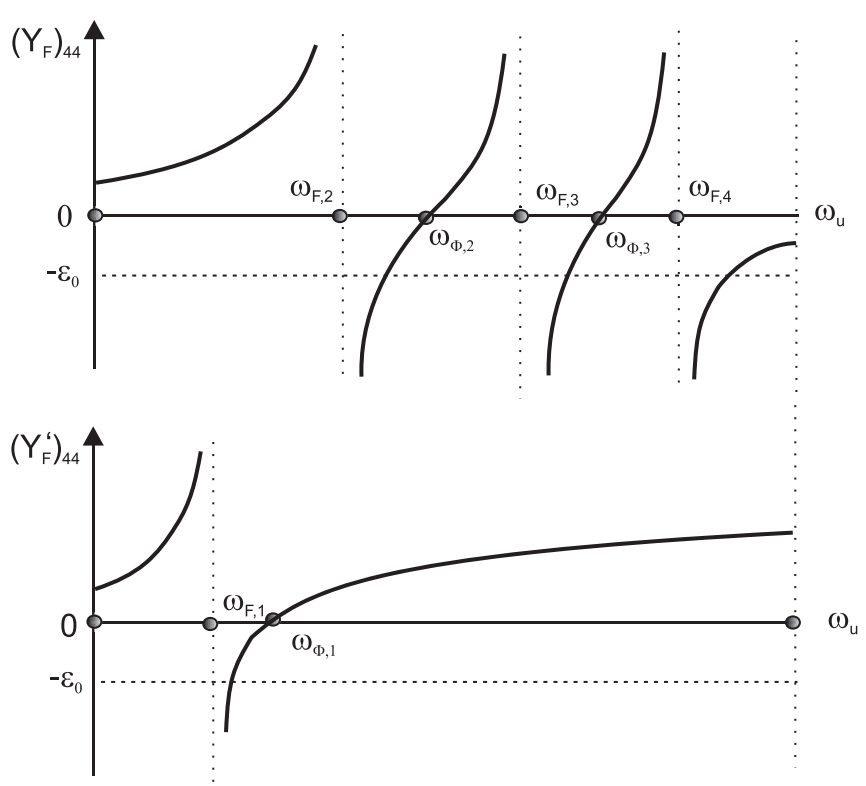

FIG. 3. Possible frequency dependencies of $Y_{F, 44}$ and $Y_{F, 44}^{\prime}$ in the lowest stop band, which ensure the existence of four SAWs in total in the direct and reversed phononic crystals bounded by vacuum.

crystallographic symmetry uncouples the piezoactive sagittally polarized SAWs (S-SAWs) and the piezoactive shear horizontally polarized SAWs (SH-SAWs).

Piezoactive S-SAWs come about when the sagittal plane spanned by the vectors $\mathbf{m}$ and $\mathbf{n}$ is the common symmetry plane for all layers of the phononic crystal [4]. For this propagation geometry, eight partial modes are split into two uncoupled sets describing six piezoactive sagittal modes and two nonpiezoactive SH modes. Correspondingly, the S-SAWs are determined through the $3 \times 3$ principal submatrices of the basic $4 \times 4$ wave matrices, such as $\hat{\mathbf{Z}}, \hat{\mathbf{Y}}$, and $\hat{\mathbf{\Xi}}_{21}$, obtained from the latter by deleting the third row and third column. The properties of a $3 \times 3$ principal submatrix ensue from those of a full $4 \times 4$ matrix. On this basis, it is straightforward to adapt the derivations and conclusions of Secs. III A and III B to the case of S-SAWs. As a result, it follows that each of the maximum possible numbers of SAWs, established under different conditions in statements (40), (41), (44), (47), and (48) with respect to the case of general anisotropy, reduces exactly by 1 when it is related to specifically the S-SAWs. In more detail, this means that (40), (41), and (44) can be adapted to predict the existence of, respectively, at most three and at most two S-SAWs under the conditions specified, while the similar adaptation of (47) and (48) predicts the existence of, respectively, at most four and at most three S-SAWs.

Consider piezoactive SH-SAWs. The waves of this type arise when the sagittal plane $(\mathbf{m}, \mathbf{n})$ is perpendicular to an even-fold symmetry axis simultaneously occurring in all layers of the phononic crystal [4]. In such a case, eight partial modes are split into two uncoupled sets describing four nonpiezoactive sagittal modes and four piezoactive SH modes. Correspondingly, the $4 \times 4$ wave matrices break up into two $2 \times 2$ diagonal blocks, the SH-SAWs being determined by the lower $2 \times 2$ block. It appropriately "inherits" the properties of the full $4 \times 4$ wave matrix. Thus, similarly as above for
S-SAWs, the general conclusions of Secs. III A and III B can be appropriately modified to establish that the maximum possible number of SH-SAWs is smaller by 2 than its value for SAWs in the general anisotropic case described by statements (40), (41), (44), (47), and (48).

Note that, in view of the modal uncoupling, the pass band/stop band spectrum splits into two independent spectra for sagittal and SH modes freely overlapping each other, so the above-mentioned statements specified differently for SSAWs and for SH-SAWs also imply correspondingly refined definitions of the sagittal or the SH full stop bands as the spectral zones where, respectively, two pairs of sagittal modes are inhomogeneous regardless of the SH modes or a pair of $\mathrm{SH}$ modes is inhomogeneous regardless of the sagittal modes. In the same spirit, it is expedient to introduce two independent sagittal and SH lowest stop bands, whose edge frequency $\omega_{u}^{(S)}$ or $\omega_{u}^{(S H)}$ is associated with the limiting wave of sagittal or $\mathrm{SH}$ polarization, respectively. Using these terms, the existence condition for SAWs on the electrically closed surface (45) can be reformulated separately for S-SAWs and for SH-SAWs in understanding that the frequency $\omega_{u}$ of the lowest stop band edge mentioned in (45) implies its different values for the sagittal and the SH modes.

\section{Mechanically clamped surface}

The condition of clamped surface, which demands zero mechanical displacement $\mathbf{a}=\mathbf{0}$ at $y=0$, is not actually met in practice. However, the knowledge about existence or nonexistence of SAW solutions on a clamped surface is important, because these solutions bring in the poles of otherwise monotonic impedance eigenvalues whose presence essentially affects the existence considerations for SAWs on the mechanically free surface or for other SAW types, e.g., such as occur in the layer-substrate structure (see Ref. [71]).

The dispersion equation for SAWs on the mechanically clamped electrically closed or open surface is $\operatorname{det}\left(\hat{\boldsymbol{\Xi}}_{P, 12}\right)=0$, see Eq. (32), and a similar equation in the case of electrically free surface is $Z_{F, 44}^{-1}=-\varepsilon_{0}$ or $Z_{F, 44}^{\prime-1}=-\varepsilon_{0}$ for the direct or the reversed phononic crystals, respectively. Altogether, the existence of SAWs on the mechanically clamped surface is determined by the matrix block $\hat{\boldsymbol{\Xi}}_{P, 12}$ of (27), in the same sense as their existence on the mechanically free surface is determined by the block $\hat{\boldsymbol{\Xi}}_{P, 21}$. In view of Eq. (38), the eigenvalues of both matrices $\hat{\mathbf{\Xi}}_{P, 12}$ and $\hat{\mathbf{\Xi}}_{P, 21}$ possess the same property of monotonicity in frequency within the full stopbands. In consequence, as far as the upper stop bands are concerned, all the statements regarding SAWs on the mechanically free surface subjected to various electrical conditions apply to SAWs on the mechanically clamped surface. A different situation, however, takes place in the lowest stop band due to dissimilar sign-definiteness properties of the eigenvalues of $\hat{\mathbf{\Xi}}_{P, 12}$ and $\hat{\mathbf{\Xi}}_{P, 21}$ specifically at $\omega=0$. According to $(43)_{2}$, all four eigenvalues of $\hat{\mathbf{\Xi}}_{F, 12}$ are negative at $\omega=0$. Hence no SAWs can occur in the lowest stop band in the case of mechanically clamped electrically open surface. In turn, since the upper diagonal $3 \times 3$ block of $\hat{\boldsymbol{\Xi}}_{\Phi, 12}$ coincides with the similar block of $\hat{\boldsymbol{\Xi}}_{F, 12}$ while the lowest diagonal component of $\hat{\boldsymbol{\Xi}}_{\Phi, 12}$ equals that of $\hat{\boldsymbol{\Xi}}_{F, 21}$, three eigenvalues of $\hat{\boldsymbol{\Xi}}_{\Phi, 12}$ are negative and one is positive at $\omega=0$. As a result, a single SAW propagating either only in the direct or only in the 
reversed phononic crystals can exist in the lowest stop band in the cases of mechanically clamped electrically closed or electrically free surface.

Note that a possible existence of a SAW on the mechanically clamped surface of a $1 \mathrm{D}$ piezoelectric phononic crystal is a specific feature in comparison with the case of SAWs in homogeneous piezoelectric substrates, where no SAWs can exist on a clamped surface for whichever anisotropy of the substrate material $[24,25]$ unless it is multiferroic, i.e., unless it possesses both piezoelectric and piezomagnetic properties $[72,73]$.

\section{NUMERICAL EXAMPLES}

With a view to provide an example of SAWs under a possibly sizable impact of piezoelectricity, we assume a phononic crystal made of layers of two materials with strong piezoelectric properties, namely, of $\mathrm{BaTiO}_{3}$ and of $0.24\left[\mathrm{~Pb}\left(\mathrm{In}_{1 / 2} \mathrm{Nb}_{1 / 2}\right) \mathrm{O}_{3}\right]-\mathrm{Pb}\left(\mathrm{Mg}_{1 / 3} \mathrm{Nb}_{2 / 3}\right) \mathrm{O}_{3}-\mathrm{PbTiO}_{3}$ (standard notation PIN24-PMN-PT). The latter belongs to a family of lead magnesium niobate-lead titanate (PMN-PT) based materials, which are widely used in actuators, sensors, and ultrasonic imaging transducers [74]. Both chosen materials are of the $4 \mathrm{~mm}$ class of crystallographic symmetry. We suppose that the crystallographic axes $X, Y$, and $Z$ of all layers are mutually aligned. The axis $Z$ is parallel to the fourfold axis. For brevity, $\mathrm{BaTiO}_{3}$ and PIN24-PMN-PT will be referred to as BTO and PPP, respectively, and a half-infinite bilayered phononic crystal will be termed PPP/BTO when the external layer is PPP, and BTO/PPP when the external layer is BTO. Regarding, e.g., PPP/BTO as the direct phononic crystal and BTO/PPP as the reversed one, we will indicate the parameters of the former and the latter without and with a prime, respectively, in agreement with the notations used throughout the paper.

The material constants of BTO are $c_{11}^{E}=275, c_{12}^{E}=179$, $c_{13}^{E}=152, \quad c_{33}^{E}=165, c_{44}^{E}=54.3, c_{66}^{E}=113$ (all in $\mathrm{GPa}$ units); $e_{31}=-2.65, e_{33}=3.64, e_{15}=21.3$ (all in $\mathrm{C} / \mathrm{m}^{2}$ units); $\varepsilon_{11}^{S} / \varepsilon_{0}=1744, \varepsilon_{33}^{S} / \varepsilon_{0}=97 ; \rho=6020 \mathrm{~kg} / \mathrm{m}^{3}$ [3]. The material constants of PPP are $c_{11}^{E}=124.3, c_{12}^{E}=109$, $c_{13}^{E}=110.2, c_{33}^{E}=124.5, c_{44}^{E}=69.8, c_{66}^{E}=62.1($ all in GPa units); $e_{31}=-9.11, e_{33}=17.6, e_{15}=8.52$ (all in $\mathrm{C} / \mathrm{m}^{2}$ units); $\varepsilon_{11}^{S} / \varepsilon_{0}=1611, \varepsilon_{33}^{S} / \varepsilon_{0}=868 ; \rho=8122 \mathrm{~kg} / \mathrm{m}^{3}$ [74]. The thickness of BTO layers will be set to be 0.75 of the thickness of PPP layers.

The dispersion curves in the following diagrams are expressed either in terms of the dimensionless frequency parameter $\omega H / v_{0}$, where $H$ is the period of phononic crystal and the quantity $v_{0}$ with a meaning of typical velocity is taken in the calculations to be $v_{0}=3000 \mathrm{~m} / \mathrm{s}$, or else in terms of the phase velocity $V=\omega / k$. Both $\omega H / v_{0}$ or $V$ are to be plotted vs the ratio $k / k_{B}$, in which $k_{B}=2 \pi / H$ is the Brillouin wave number.

Let us exemplify piezoactive S-SAWs. For that, we assume that the normal $\mathbf{n}$ to the plane of layer interfaces lies in the common symmetry plane $Z X$ of layers and we take the propagation direction $\mathbf{m}$ in this plane so that the sagittal plane $(\mathbf{m}, \mathbf{n})$ turns out to be a symmetry plane thus ensuring uncoupling of the piezoactive sagittal modes. In addition, we take the normal $\mathbf{n}$ to be parallel to the $Z$ axis.

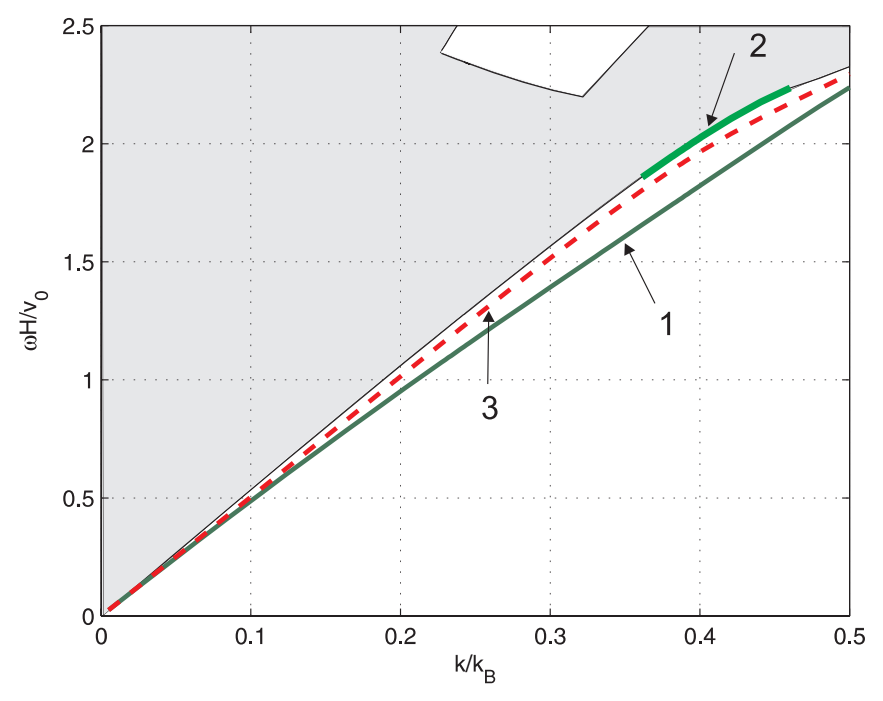

FIG. 4. Frequency vs wave-number dispersion branches of SSAWs in the lowest stop band of PPP/BTO and BTO/PPP phononic crystals with mechanically free electrically closed surface. Grey region: the first pass band of sagittal waves. White regions: full stop bands of sagittal waves. Curve 1: slow S-SAW with frequency $\omega_{c, 1}$ in PPP/BTO; curve 2: fast S-SAW with frequency $\omega_{c, 2}$ in PPP/BTO; curve 3: S-SAW with frequency $\omega_{c}^{\prime}$ in BTO/PPP.

Figure 4 shows the frequency vs wave-number S-SAW dispersion branches in the lowest stop band of PPP/BTO (curves 1 and 2) and BTO/PPP (curve 3) phononic crystals with mechanically free electrically closed surface. It is seen that faster among two S-SAWs for PPP/BTO occurs only within a narrow interval of wave number $k$ values and that the frequency $\omega_{c, 2}(k)$ of this S-SAW is only slightly less than the stop band edge frequency $\omega_{u}(k)$ (i.e., the frequency of the sagittal limiting wave; see Sec. III C). For the same stop band, the velocity vs wave-number curves of S-SAWs both on electrically closed and the electrically open surfaces are depicted separately for PPP/BTO and BTO/PPP in Figs. 5 and 6 , respectively. Figure 5 also demonstrates the dispersion dependence of the velocity $V_{u}=\omega_{u} / k$ of the sagittal limiting wave and Fig. 6 reproduces the same curve as a benchmark.

It follows from Figs. 4-6 that the mechanically free electrically closed surface of PPP/BTO supports two S-SAWs in the lowest stop band and that one more S-SAW exists on this surface in the case of BTO/PPP. In turn, one S-SAW occurs in the same stop band for each phononic crystal with mechanically free electrically open surface. Thus, in view of the results of Sec. IIIC, the total number of S-SAWs, which embraces their occurrences in the direct and reversed phononic crystals PPP/BTO and BTO/PPP, is the maximum possible for the lowest stop band in both cases of electrically closed and electrically open boundary conditions. We have also checked that there is one S-SAW in this stop band for each of the PPP/BTO and BTO/PPP phononic crystals with electrically free surface. In fact, the velocities of these S-SAWs are hardly different from the velocities of S-SAWs on the electrically open surface because of the very large permittivity of BTO and PPP.

It can be verified that the S-SAW velocities $V_{c, 1}$ and $V_{c}^{\prime}$ transform, respectively, to the velocities $V_{o, 1}$ and $V_{o}^{\prime}$ (see 


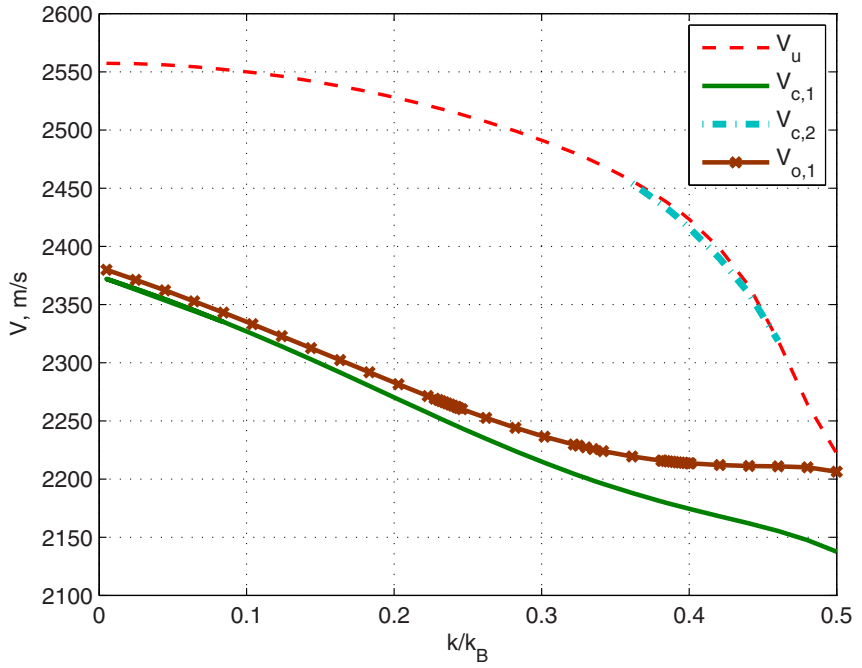

FIG. 5. Velocity vs wave-number dispersion branches of SSAWs in the lowest stop band of PPP/BTO phononic crystal with electrically closed and electrically open surface. $V_{c, 1}$ : slow S-SAW velocity on the electrically closed surface; $V_{c, 2}$ : fast S-SAW velocity on the electrically closed surface; $V_{o}$ : S-SAW velocity on the electrically open surface; $V_{u}$ : velocity of the sagittal limiting wave.

Figs. 4 and 5), as the dielectric permittivity of the exterior space changes from $\infty$ (electrically closed surface) to 0 (electrically open surface). Recall that in the case of a purely elastic phononic crystal with any asymmetric (in particular, bilayered) arrangement of unit cell the maximum possible number of S-SAWs per full stop band for direct and reversed phononic crystals in total is 2 [64]. Hence one can interpret the existence of two piezoactive S-SAWs of the aforementioned type as being primarily due to the elastic properties of materials, while the piezoelectric effect only brings in some corrections to these S-SAWs. Correspondingly,

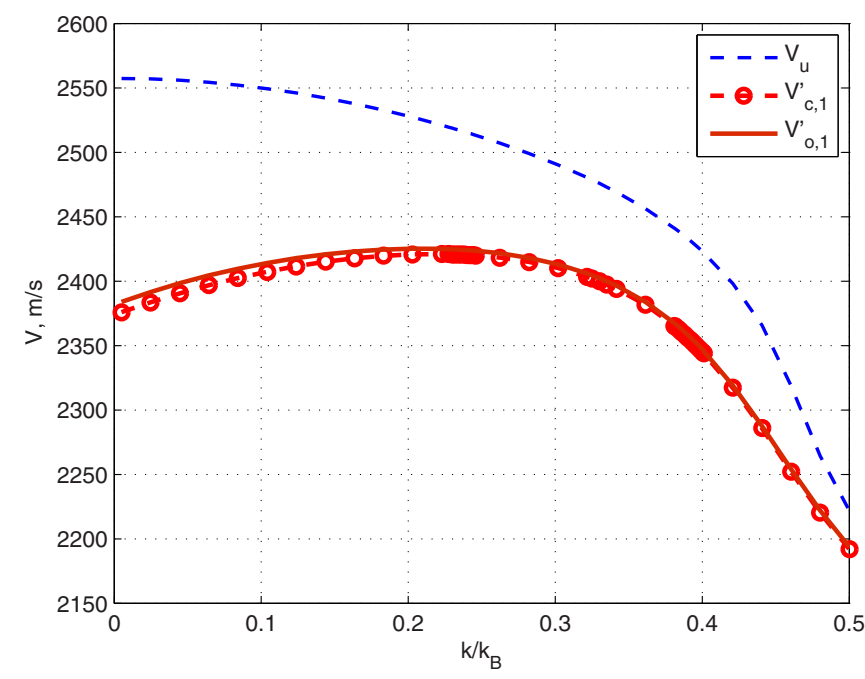

FIG. 6. Velocitiy vs wave-number dispersion branches of SSAWs in the lowest stop band of BTO/PPP phononic crystal with electrically closed and electrically open surface. $V_{c}^{\prime}$ : S-SAW velocity on the electrically closed surface; $V_{o}^{\prime}:$ S-SAW velocity on the electrically open surface; $V_{u}$ : velocity of the sagittal limiting wave.

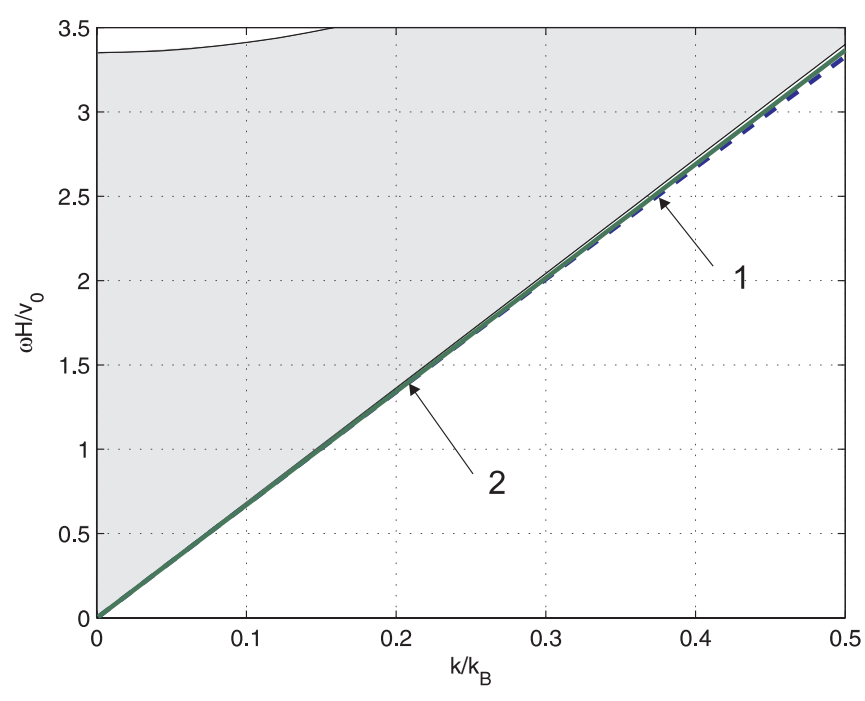

FIG. 7. Frequency vs wave number of SH-SAWs in the lowest stop band of PPP/BTO and BTO/PPP phononic crystals with mechanically free electrically closed surface. Grey region: the first pass band of SH waves. White regions: full stop bands of SH waves. Curve 1: S-SAW with frequency $\omega_{c}$ in PPP/BTO; curve 2: S-SAW with frequency $\omega_{c}^{\prime}$ in BTO/PPP.

the occurrence of a faster S-SAW on the electrically closed surface of PPP/BTO can be perceived as being caused entirely by the piezoelectricity.

Consider now piezoactive SH-SAWs in PPP/BTO and BTO/PPP phononic crystals. To model this case, we assume that the plane of layer interfaces contains the fourfold symmetry axis, i.e., the crystallographic $Z$ axis, of the constituent layers, hence taking the propagation direction $\mathbf{m}$ orthogonal to this axis provides uncoupling of the piezoactive $\mathrm{SH}$ modes. We also assume that the plane of layer interfaces coincides with the symmetry plane $Z X$ of the layers.

Figure 7 shows the frequency vs wave-number SH-SAW dispersion branches in the lowest stop band of PPP/BTO and BTO/PPP phononic crystals with mechanically free electrically closed surface. The corresponding velocity vs wave-number dispersion branches on the electrically closed and on the electrically open surface of PPP/BTO and $\mathrm{BTO} / \mathrm{PPP}$ along with the dispersion dependence of the $\mathrm{SH}$ limiting wave velocity $V_{u}$ are displayed in Fig. 8. There is one SH-SAW for each of two PPP/BTO and BTO/PPP phononic crystals in the case of electrically closed surface, and there is one SH-SAW for PPP/BTO in the case of electrically open surface. Thus, according to Sec. IIIC, in both these cases the PPP/BTO and BTO/PPP phononic crystals support the maximum possible number of SH-SAWs in the lowest stop band. If the surface is bounded by vacuum, then the SH-SAW exists only in PPP/BTO. Its velocity practically coincides with that on the electrically open surface.

According to Ref. [64], the lowest stop band of a purely elastic half-infinite phononic crystal with a bilayered unit cell always contains one and only one frequency of SH-SAW occurring in either the direct or the reversed phononic crystals. Therefore, one of the two piezoactive SH-SAWs observed in PPP/BTO and BTO/PPP phononic crystals with electrically closed surface comes about entirely due to the piezoelectric 


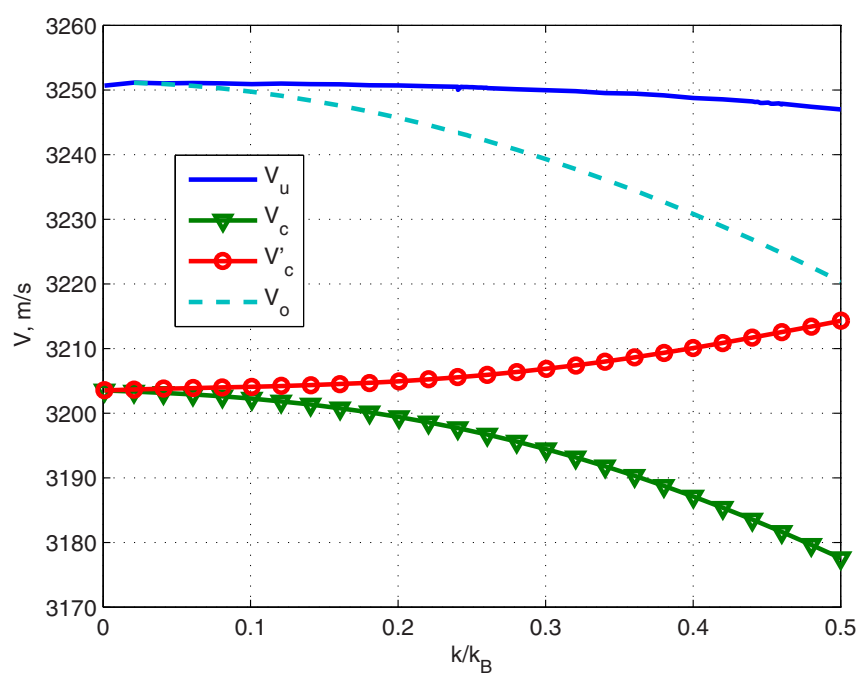

FIG. 8. Velocity vs wave-number dispersion curves of SH-SAWs in the lowest stop band of PPP/BTO and BTO/PPP phononic crystals with electrically closed surface and with electrically open surface. $V_{c}$ : SH-SAW velocity on the electrically closed surface of PPP/BTO; $V_{c}^{\prime}$ : SH-SAW velocity on the electrically closed surface of BTO/PPP; $V_{o}$ : SH-SAW velocity on the electrically open surface of PPP/BTO; $V_{u}$ : velocity of the SH limiting wave.

effect. On purely analytical grounds, it is impossible to determine which of these two waves is "piezoelectricity borne." However, taking into account the fact that the SHSAW in the lowest stop band exists in PPP/BTO for both cases of electrically closed and electrically open surfaces, one can conjecture that it is the SH-SAW in BTO/PPP which occurs due to the piezoelectric effect.

No SAWs can exist in the lowest stop band of a purely elastic half-infinite phononic crystal if its surface is mechanically clamped [64]. In contrast, the lowest stop band of a piezoelectric phononic crystal admits

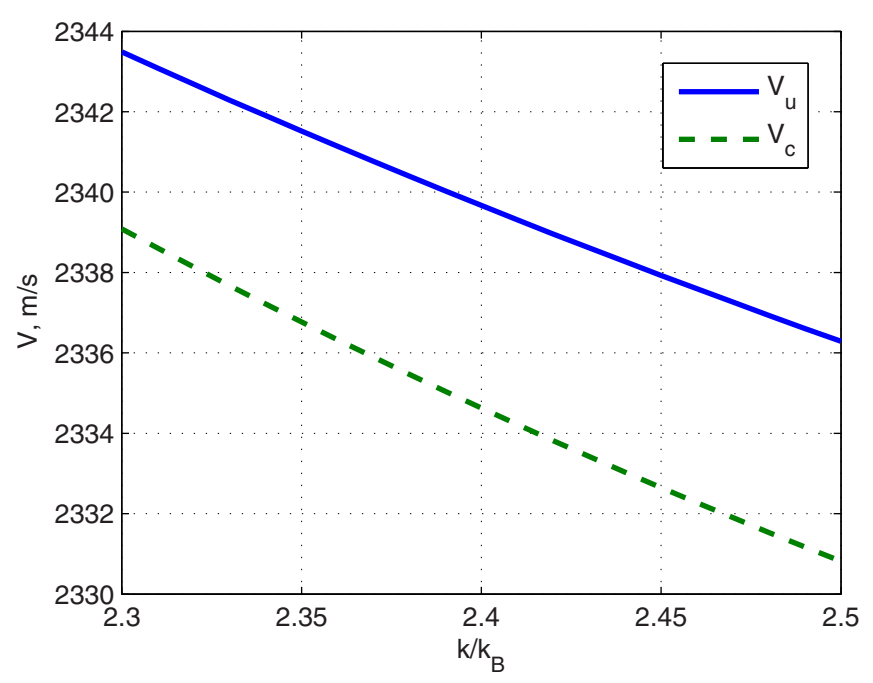

FIG. 9. Velocity vs wave-number dispersion curves of SH-SAWs in the lowest stop band of PIN-PMN-PT:Mn-BTO phononic crystal with the mechanically clamped electrically closed surface. $V_{c}: \mathrm{SH}-$ SAW velocity; $V_{u}$ : velocity of the $\mathrm{SH}$ limiting wave. the existence of one SAW on the mechanically clamped electrically closed or electrically free surface. Let us give an example of such a wave in the case of an electrically closed surface. Consider a periodically bilayered phononic crystal formed of $\mathrm{BaTiO}_{3}$ (BTO) and of $\mathrm{Mn}$ modified $0.26 \mathrm{~Pb}\left(\mathrm{In}_{1 / 2} \mathrm{Nb}_{1 / 2}\right) \mathrm{O}_{3}-0.42 \mathrm{~Pb}\left(\mathrm{Mg}_{1 / 3} \mathrm{Nb}_{2 / 3}\right) \mathrm{O}_{3}-0.32 \mathrm{PbTiO}_{3}$ (standard notation PIN-PMN-PT:Mn). Let the exterior layer be of the latter material. Its crystallographic symmetry is $m m 2$ and the material constants are as follows: $c_{11}^{E}=200, \quad c_{12}^{E}=106, \quad c_{13}^{E}=46.4, \quad c_{22}^{E}=149, \quad c_{23}^{E}=102$, $c_{33}^{E}=150, c_{44}^{E}=18.4, c_{55}^{E}=4.9, c_{66}^{E}=71.4$ (all in $\mathrm{GPa}$ units); $\quad e_{15}=15.2, \quad e_{24}=44.8, \quad e_{31}=2.38, \quad e_{32}=-6.23$, $e_{33}=14.5$ (all in $\mathrm{C} / \mathrm{m}^{2}$ units); $\varepsilon_{11}^{S} / \varepsilon_{0}=679, \varepsilon_{22}^{S} / \varepsilon_{0}=4672$, $\varepsilon_{33}^{S} / \varepsilon_{0}=561, \rho=8120 \mathrm{~kg} / \mathrm{m}^{-3}$ [75]. The thickness of BTO layers is set as 0.3 of the thickness of PIN-PMN-PT:Mn layers. It is assumed that the fourfold symmetry axes of BTO layers and the twofold symmetry axes of the PIN-PMN-PT:Mn layers are mutually aligned and that their symmetry planes are also parallel to each other and coincide with the plane of layer interfaces. Given this setting, the propagation direction orthogonal to the joint symmetry axis uncouples the piezoactive $\mathrm{SH}$ modes. Figure 9 depicts a part of the SH-SAW dispersion branch occurring in the lowest stop band curve of the given phononic crystal in the case of its mechanically clamped electrically closed surface.

\section{CONCLUDING REMARKS}

The present paper has studied the existence of SAWs in 1D half-infinite piezoelectric phononic crystals of arbitrary anisotropy. This framework generally precludes finding explicit expressions for the acoustoelectric wave fields. Instead, we use particular intrinsic symmetries of the unit-cell transfer matrix in conjunction with certain sign-definiteness properties of the surface impedance and admittance matrices, which altogether allow us to establish the maximum possible number of SAWs occurring per full stop band in total in the direct and reverse phononic crystals. This number depends on the type of electrical conditions supplementing the traction-free condition on a crystal surface. There is also a difference between the number of SAWs in the lowest stop bands, which extend from zero frequency, and in the other full stop bands. The difference is due to the fact that the admittance and impedance matrices at $\omega=0$ possess some specific sign-definiteness properties, which are missing at the lower edge of the other full stopbands. The results obtained for the case of arbitrary anisotropy of constituting layers have also been specialized for the symmetry-based cases of sagittal and SH piezoactive SAWs. The overall main conclusions are summarized in Table I. Besides these, the SAWs on a mechanically clamped surface were briefly considered as well.

Comparing the results of the present paper with those obtained for purely elastic 1D phononic crystals in Ref. [64] shows that the piezoelectric coupling increases the possible number of SAWs, i.e., it allows new types of SAWs not existing in the purely elastic case. Of course, the emergence of additional SAWs is only a possibility, which comes into being provided the piezoelectric effect is strong enough. However, the only restriction on the piezoelectric coupling is supposed to come from the condition of medium thermodynamic 
TABLE I. Maximum total number of SAWs per full stop band at a fixed $k$ in the direct and reversed phononic crystals with different boundary conditions on the mechanically free surface. Notation in the first column is $e$ closed: electrically closed surface; $e$ open: electrically open surface; $e$ free: boundary with vacuum. Other columns refer to the SAWs in the case of general anisotropy, to the piezoactive S-SAWs, and to the piezoactive SH-SAWs. The notation $\left(0, \omega_{u}\right)$ implies lowest stop band and $\left(\omega_{l}, \omega_{u}\right)$ implies upper stop bands.

\begin{tabular}{|c|c|c|c|c|c|c|}
\hline \multirow[b]{2}{*}{ Surface } & \multicolumn{2}{|c|}{ SAWs } & \multicolumn{2}{|c|}{ S-SAWs } & \multicolumn{2}{|c|}{ SH-SAWs } \\
\hline & $\left(0, \omega_{u}\right)$ & $\left(\omega_{l}, \omega_{u}\right)$ & $\left(0, \omega_{u}\right)$ & $\left(\omega_{l}, \omega_{u}\right)$ & $\left(0, \omega_{u}\right)$ & $\left(\omega_{l}, \omega_{u}\right)$ \\
\hline$e$ closed & 4 & 4 & 3 & 3 & 2 & 2 \\
\hline$e$ open & 3 & 4 & 2 & 3 & 1 & 2 \\
\hline$e$ free & 4 & 5 & 3 & 4 & 2 & 3 \\
\hline
\end{tabular}

stability and this does not impose direct upper bounds upon the piezoelectric coefficients. Therefore the occurrence of such additional piezoelectricity-stipulated SAWs in 1D phononic crystals is perfectly feasible in practice. It has been numerically modeled in Sec. IV.

Note a possible generalization of the present results to the case of 1D functionally graded phononic crystals. For such media, the transfer matrix is no longer a product of a finite number of matrix exponentials but it is a so-called multiplicative integral of the system matrix; see [68]. This fact certainly makes the explicit calculations more intricate; however, all the conclusions on the SAW existence obtained herein for layered periodic phononic crystals apply as well to functionally graded periodic materials.

Finally let us point out two issues, which have not been discussed in this paper but which seem quite worthy of further study. First, we note that the above-presented results, which are valid for any ordering of layers within a period of a phononic crystal, can be substantially specialized for the case where this ordering is arranged to be symmetric with respect to the period midplane. In this case, the unit-cell transfer matrix $\hat{\mathbf{M}}_{P}$ acquires certain additional algebraic symmetry which should restrict the maximum possible number of SAWs, like it was established for the case of purely elastic phononic crystals in Ref. [64]. Second, the case of SAWs propagating normally to the layer interfaces also deserves a special attention. It can be shown that a zero tangential wave number $k$ modifies the general predictions on the SAW existence. Both these issues along with some other topics of interest will be considered elsewhere.

\section{ACKNOWLEDGMENTS}

The authors thank V. I. Alshits and A. V. Sarychev for helpful discussions. The work by A. Darinskii was supported by the Ministry of Science and Higher Education of the Russian Federation within the State assignment FSRC "Crystallography and Photonics" RAS.

\section{APPENDIX}

Assume a 1D half-infinite phononic crystal $y>0$ and consider a wave of the type (3) in there, which propagates within a full stop band and vanishes at the infinite depth. According to [65], its time average local kinetic energy per unit surface can be cast in the form

$$
\begin{aligned}
E(y) & =\frac{\rho \omega^{2}}{4}|\mathbf{u}|^{2}=-\frac{\omega k^{2}}{8} \xi_{P}^{\dagger}\left(\frac{\partial}{\partial \omega} \hat{\mathbf{T}} \hat{\mathbf{N}}_{P}\right) \xi_{P} \\
& =\frac{i \omega k}{8} \frac{d}{d y}\left(\xi_{P}^{\dagger} \hat{\mathbf{T}} \frac{\partial \xi_{P}}{\partial \omega}\right),
\end{aligned}
$$

where $\xi_{P}(y)$ is the solution of Eq. (6) referred to the $m$ th layer. Note that $E$ certainly remains nonzero at $k=0$ because of the factor $k^{-1}$ in the definition of $\mathbf{I}$ and $d$, which enter $\xi_{P}$; see Eq. (4). In view of Eq. (A1), the continuity of the vector $\xi_{P}(y)$ at the layer interfaces, and its zero limit at $y \rightarrow \infty$, the total kinetic energy per unit surface $\bar{E}=\int_{0}^{\infty} E(y) d y$ reduces to a contribution at the surface $y=0$, namely

$$
\bar{E}=-\frac{i \omega k}{8}\left(\xi_{P}^{\dagger} \hat{\mathbf{T}}^{\frac{\partial \xi_{P}}{\partial \omega}}\right)_{y=0}=-\frac{i \omega k}{8}\left(\mathbf{U}_{P}^{\dagger} \frac{\partial \mathbf{V}_{P}}{\partial \omega}+\mathbf{V}_{P}^{\dagger} \frac{\partial \mathbf{U}_{P}}{\partial \omega}\right),
$$

where $\xi_{P}=\sum_{\alpha=1}^{4} b_{\alpha} \zeta_{P, \alpha}$ and hence $\mathbf{U}_{P}=\sum_{\alpha=1}^{4} b_{\alpha} \mathbf{U}_{P, \alpha}$, $\mathbf{V}_{P}=\sum_{\alpha=1}^{4} b_{\alpha} \mathbf{V}_{P, \alpha}$, according to the adopted numbering of those four eigenvectors $\zeta_{P, \alpha}$ of $\hat{\mathbf{M}}_{P}$ which generate the decaying partial modes; see Sec. II A. By (A2), recalling definition (33) of the admittance for the direct phononic crystal and assuming that either the vector $\mathbf{V}_{P}$ or the vector $\mathbf{U}_{P}$ does not depend on frequency, we obtain, respectively,

$$
\bar{E}=\frac{\omega k}{8} \mathbf{V}_{P}^{\dagger} \frac{\partial \hat{\mathbf{Y}}_{P}}{\partial \omega} \mathbf{V}_{P} \quad \text { or } \quad \bar{E}=-\frac{\omega k}{8} \mathbf{U}_{P}^{\dagger} \frac{\partial \hat{\mathbf{Z}}_{P}}{\partial \omega} \mathbf{U}_{P} .
$$

In the case of reversed phononic crystal $y<0$, the total kinetic energy $\bar{E}^{\prime}=\int_{-\infty}^{0} E(y) d y$ satisfies Eq. (A2) with the opposite sign on the right-hand side, which is then inverted once more due to the opposite signs in the impedance definitions (33) for the direct and reversed phononic crystal. Hence

$$
\bar{E}^{\prime}=\frac{\omega k}{8} \mathbf{V}_{P}^{\prime \dagger} \frac{\partial \hat{\mathbf{Y}}_{P}^{\prime}}{\partial \omega} \mathbf{V}_{P}^{\prime} \text { or } \bar{E}^{\prime}=-\frac{\omega k}{8} \mathbf{U}_{P}^{\prime \dagger} \frac{\partial \hat{\mathbf{Z}}_{P}^{\prime}}{\partial \omega} \mathbf{U}_{P}^{\prime}
$$

under the condition that, respectively, either $\mathbf{V}_{P}^{\prime}=$ $\sum_{\alpha=5}^{8} b_{\alpha}^{\prime} \mathbf{V}_{P, \alpha}$ or $\mathbf{U}_{P}^{\prime}=\sum_{\alpha=5}^{8} b_{\alpha}^{\prime} \mathbf{U}_{P, \alpha}$ does not depend on frequency. Note that the admittances and impedances are Hermitian matrices and that $\mathbf{U}_{P}$ or $\mathbf{V}_{P}$ and $\mathbf{U}_{P}^{\prime}$ or $\mathbf{V}_{P}^{\prime}$ are arbitrary constant vectors (i.e., independent of frequency which can always be arranged via the coefficients $b_{\alpha}$ or $\left.b_{\alpha}^{\prime}\right)$. Thus, since the kinetic energy is a positive quantity, Eqs. (A3) and (A4) reveal the corresponding sign-definiteness of frequency derivatives of the admittance and impedance matrices, which is expressed in Eqs. (36) and (37).

Now consider the time average local internal energy per unit surface,

$$
W(y)=\frac{1}{8}\left(\sigma_{i j} \frac{\partial u_{i}^{*}}{\partial x_{j}}-\frac{\partial \varphi}{\partial x_{j}} D_{j}^{*}+\sigma_{i j}^{*} \frac{\partial u_{i}}{\partial x_{j}}-\frac{\partial \varphi^{*}}{\partial x_{j}} D_{j}\right),
$$

associated with the wave (3) in the $m$ th layer. In the static limit, by virtue of Eq. (1), the right-hand side of Eq. (A5) taken at $\omega=0$ can be presented as a full divergence, which actually reduces to a derivative in the thickness coordinate $x_{2} \equiv y$, so that

$$
\left.W(y)\right|_{\omega=0}=\frac{1}{8} \frac{d}{d y}\left(\sigma_{i 2} u_{i}^{*}-\varphi D_{2}^{*}+\sigma_{i 2}^{*} u_{i}-\varphi^{*} D_{2}\right) .
$$


Thus, assuming a half-infinite phononic crystal $y>0$ and introducing the total internal energy of the wave (3) per unit surface $\bar{W}=\int_{0}^{\infty} W(y) d y$, we observe from Eq. (A6) that the static limit of $\bar{W}$ can be evaluated by exact analogy with the above instance of the kinetic energy. Recall that, for any $k \neq 0$, a certain vicinity of zero frequency is always a full stop band (see Sec. II A) and so the static limit of the wave (3) can be assumed in the form of a four-partial superposition of the modes $\alpha=1, \ldots, 4$ vanishing at the infinite depth. Using this fact and invoking Hermiticity of the impedance and admittance matrices, we obtain

$$
\begin{aligned}
\left.\bar{W}\right|_{\omega=0} & =-\frac{1}{8}\left(\sigma_{i 2} u_{i}^{*}-\varphi D_{2}^{*}+\sigma_{i 2}^{*} u_{i}-\varphi^{*} D_{2}\right)_{y=0} \\
& =\frac{i k}{8}\left(\mathbf{U}_{F}^{\dagger} \mathbf{V}_{F}-\mathbf{V}_{F}^{\dagger} \mathbf{U}_{F}\right)=\frac{k}{4} \mathbf{V}_{F}^{\dagger} \hat{\mathbf{Y}}_{F} \mathbf{V}_{F}=\frac{k}{4} \mathbf{U}_{F}^{\dagger} \hat{\mathbf{Z}}_{F} \mathbf{U}_{F},
\end{aligned}
$$

where $\mathbf{U}_{F}$ and $\mathbf{V}_{F}$ are defined as in (A2). An analogous relation for the total internal energy $\bar{W}^{\prime}=\int_{-\infty}^{0} W(y) d y$ in the reversed phononic crystal $y<0$ follows in the form

$$
\left.\bar{W}^{\prime}\right|_{\omega=0}=\frac{k}{4} \mathbf{V}_{F}^{\prime \dagger} \hat{\mathbf{Y}}_{F}^{\prime} \mathbf{V}_{F}^{\prime}=\frac{k}{4} \mathbf{U}_{F}^{\prime \dagger} \hat{\mathbf{Z}}_{F}^{\prime} \mathbf{U}_{F}^{\prime},
$$

where $\mathbf{V}_{F}^{\prime}$ and $\mathbf{U}_{F}^{\prime}$ are defined as in (A4). Since the internal energy is positive, Eqs. (A7) and (A8) imply positive-definiteness of the static limit of admittance and impedance matrices, which is expressed in Eq. (42).

The admittances in $\Phi$ representation are related at $\omega=0$ to the electric enthalpy, the appropriate expressions being similar to Eqs. (A7) and (A8). The electric enthalpy is not sign-definite [1,3], neither are $\hat{\mathbf{Y}}_{\Phi}$ and $\hat{\mathbf{Y}}_{\Phi}^{\prime}$.
[1] Yu. I. Sirotin and M. P. Shaskol'skaya, Fundamentals of Crystal Physics (Mir, Moscow, 1982).

[2] J. F. Nye, Physical Properties of Crystals (Oxford University Press, Oxford, 1985).

[3] J. Yang, An Introduction to the Theory of Piezoelectricity (Springer Science + Business Media, Inc., Boston, 2005).

[4] B. A. Auld, Acoustic Fields and Waves in Solids (Krieger, Malabar, FL, 1990), Vols. I and II.

[5] G. A. Maugin, Continuum Mechanics of Electromagnetic Solids (North-Holland, Amsterdam, 1988).

[6] A. R. Hutson, J. H. McFree, and D. L. White, Ultrasonic Amplification in CdS, Phys. Rev. Lett. 7, 237 (1961).

[7] D. L. White, Amplification of ultrasonic waves in piezoeelctric semiconductors, J. Appl. Phys. 33, 2547 (1962).

[8] J. H. McFee, Transmission and amplification of acoustic waves in piezoelectric semiconductors, in Physical Acoustics, edited by W. P. Mason (1966), Vol. V.4A, pp. 1-44.

[9] V. I. Pustovoit, Interaction of electron streams with elastic lattice waves, Sov. Phys. Usp. 12, 105 (1969) [Usp. Fiz. Nauk 97, 257 (1969)].

[10] G. Rozas, M. F. Pascual Winter, A. Fainstein, B. Jusserand, P. O. Vaccaro, S. Saravanan, and N. Saito, Piezoelectric semiconductor acoustic cavities, Phys. Rev. B 72, 035331 (2005).

[11] B. A. Glavin, V. A. Kochelap, T. L. Linnik, A. J. Kent, N. M. Stanton, and M. Henini, Resonance-like piezoelectric electronphonon interaction in layered structures, Phys. Rev. B 74, 165317 (2006).

[12] V. J. Gokhale and M. Rais-Zadeh, Phonon-electron interactions in piezoelectric semiconductor bulk acoustic wave resonators, Sci. Rep. 4, 5617 (2014).

[13] M. Willatzen and J. Christensen, Acoustic gain in piezoelectric semiconductors at $\varepsilon$-near-zero response, Phys. Rev. B 89, 041201(R) (2014).

[14] RF Bulk Acoustic Wave Filters for Communications, edited by K.-Y. Hashimoto (Artech House, Norwood, 2009).

[15] Y.-y. Zhu, X.-j. Zhang, Y.-q. Lu, Y.-f. Chen, S.-n. Zhu, and N. Ming, New Type of Polariton in a Piezoelectric Superlattice, Phys. Rev. Lett. 90, 053903 (2003).
[16] X.-j. Zhang, R.-q. Zhu, J. Zhao, Y.-f. Chen, and Y.-y. Zhu, Phonon-polariton dispersion and the polariton-based photonic band gap in piezoelectric superlattices, Phys. Rev. B 69, 085118 (2004)

[17] Z. Liu and W. Zhang, Scaling and localization of polaritonic states in piezoelectric Fibonacci superlattices, Phys. Rev. B 72, 134304 (2005).

[18] Y. Chao, J. Sheng, J. A. Sedlacek, and J. P. Shaffer, Surface phonon polaritons on anisotropic piezoelectric superlattices, Phys. Rev. B 93, 045419 (2016).

[19] D. Morgan, Surface Acoustic Wave Filters (Elsevier, New York, 2007).

[20] S. Völk, A. Wixforth, D. Reuter, A. D. Wieck, and J. Ebbecke, Conversion of bound excitons to free excitons by surface acoustic waves, Phys. Rev. B 80, 165307 (2009).

[21] M. Prunnila and J. Meltaus, Acoustic Phonon Tunneling and Heat Transport due to Evanescent Electric Fields, Phys. Rev. Lett. 105, 125501 (2010).

[22] K. A. Ingebrigtsen, Surface waves in piezoelectrics, J. Appl. Phys. 40, 2681 (1969).

[23] E. A. Kraut, New mathematical formulation for piezoelectric wave propagation, Phys. Rev. 188, 1450 (1969).

[24] J. Lothe and D. M. Barnett, Integral formalism for surface waves in piezoelectric crystals. Existence considerations, J. Appl. Phys. 47, 1799 (1976).

[25] J. Lothe and D. M. Barnett, Further development of the theory for surface waves in piezoelectric crystals, Phys. Norvegica 8 , 239 (1977).

[26] R. C. Peach, On the existence of surface acoustic waves on piezoelectric substrates, IEEE Transactions on Ultrasonics, IEEE Trans. Ultrason. Ferroelectr. Freq. Control 48, 1308 (2001).

[27] J. Lothe and D. M. Barnett, On the existence of surface wave solutions for anisotropic half-spaces with free surface, J. Appl. Phys. 47, 428 (1976).

[28] D. M. Barnett and J. Lothe, Free surface (Rayleigh) waves in anisotropic media: The surface impedance method, Proc. R. Soc. London, Ser. A 402, 135 (1985). 
[29] M. Abbudi and D. M. Barnett, On the existence of interfacial (Stoneley) waves in bonded piezoelectric half-spaces, Proc. R. Soc. London, Ser. A 429, 587 (1990).

[30] V. I. Alshits, D. M. Barnett, A. N. Darinskii, and J. Lothe, On the existence problem for localized acoustic waves on the interface between two piezocrystals, Wave Motion 20, 233 (1994).

[31] E. H. El Boudouti, B. Djafari-Rouhani, A. Akjouj, and L. Dobrzynski, Acoustic waves in solid and fluid layered materials, Surf. Sci. Rep. 64, 471 (2009).

[32] Acoustic Metamaterials and Phononic Crystals, edited by P. A. Deymier (Springer, Berlin, 2013).

[33] V. Laude, Phononic Crystals: Artificial Crystals for Sonic, Acoustic, and Elastic Waves, Studies in Mathematical Physics No. 26 (De Gruyter, 2015).

[34] Phononic Crystals. Fundamentals and Applications, edited by A. Khelif and A. Adibi (Springer, New York, 2016).

[35] E. H. El Boudouti and B. Djafari-Rouhani, One-Dimensional Phononic Crystals, in Acoustic Metamaterials and Phononic Crystals, edited by P. A. Deymier (Springer, Berlin, 2013), p. 45.

[36] R. E. Camley, B. Djafari-Rouhani, L. Dobrzynski, and A. A. Maradudin, Transverse elastic waves in periodically layered infinite and semi-infinite media, Phys. Rev. B 27, 7318 (1983).

[37] A. Nougaoui and B. Djafari-Rouhani, Dynamics of infinite and semi-infinite piezoelectric superlattices: Shear horizontal waves and effective medium approximation, Surf. Sci. 185, 154 (1987).

[38] E. H. El Boudouti, B. Djafari-Rouhani, E. M. Khourdifi, and L. Dobrzynski, Surface and interface elastic waves in superlattices: transverse localized and resonant modes, Phys. Rev. B 48, 10987 (1993).

[39] E. H. El Boudouti, B. Djafari-Rouhani, A. Akjouj, and L. Dobrzynski, Theory of surface and interface transverse elastic waves in $N$-layer superlattices, Phys. Rev. B 54, 14728 (1996).

[40] A. Bousfia, E. H. El Boudouti, D. Bria, and A. Nougaoui, Surface and interface shear horizontal acoustic waves in piezoelectric superlattices, J. Appl. Phys. 87, 4507 (2000).

[41] S.-Y. Ren and Y.-C. Chang, Theory of confinement effects in finite one-dimensional phononic crystals, Phys. Rev. B 75, 212301 (2007).

[42] S. Chen, S. Lin, and Z. Wong, Shear horizontal surface acoustic waves in semi-infinite piezoelectrics/metal superlattices, Ultrasonics 49, 446 (2009).

[43] Ph. Gatignol, C. Potel, and J. F. de Belleval, Two families of modal waves for periodic structures with two field functions: a Cayleigh-Hamilton approach, Acust. Acta Acust. 93, 959 (2007).

[44] A. L. Shuvalov, O. Poncelet, and S. V. Golkin, Existence and spectral properties of shear horizontal surface waves in vertically periodic half-spaces, Proc. R. Soc. London, Ser. A 465, 1489 (2009).

[45] M. Alami, E. H. El Boudouti, B. Djafari-Rouhani, Y. El Hassouani, and A. Talbi, Surface acoustic waves in onedimensional piezoelectric-metallic phononic crystal: Effect of a cap layer, Ultrasonics 90, 80 (2018).

[46] B. Djafari-Rouhani, L. Dobrzynski, O. Hardouin Duparc, R. E. Camley, and A. A. Maradudin, Sagittal elastic waves in infinite and semi-infinite superlattices, Phys. Rev. B 28, 1711 (1983).
[47] A. Nougaoui and B. Djafari-Rouhani, Elastic waves in periodically layered infinite and semi-infinite anisotropic media, Surf. Sci. 185, 125 (1987).

[48] E. H. El Boudouti, B. Djafari-Rouhani, and A. Nougaoui, Surface elastic waves in superlattices: Sagittal localized and resonant modes, Phys. Rev. B 51, 13801 (1995).

[49] A. Nougaoui and B. Djafari-Rouhani, Vibrations in elastic and piezoelectric superlattices, J. Electron. Spectrosc. Relat. Phenom. 45, 197 (1987).

[50] S. Chen, Y. Zhang, S. Lin, and Z. Fu, Study on the electromechanical coupling coefficient of Rayleigh-type surface acoustic waves in semi-infinite piezoelectrics/non-piezoelectrics superlattices, Ultrasonics 54, 604 (2014).

[51] A. Nougaoui and B. Djafari-Rouhani, Complex band structure of acoustic waves in superlattices, Surf. Sci. 199, 623 (1988).

[52] B. Honein, A. M. B. Braga, P. Barbone, and G. Herrmann, Wave Propagation in Piezoelectric Layered Media with Some Applications, J. Intell. Mater. Syst. Struct. 2, 542 (1991).

[53] A. M. B. Braga and G. Herrmann, Floquet waves in anisotropic periodically layered composites, J. Acoust. Soc. Am. 91, 1211 (1992).

[54] T. Aono and S. Tamura, Surface and pseudosurface acoustic waves in superlattices, Phys. Rev. B 58, 4838 (1998).

[55] Y. El Hassouani, E. H. El Boudouti, B. Djafari-Rouhani, H. Aynaou, and L. Dobrzynski, Surface and interface acoustic waves in solid-fluid superlattices: Green's function approach, Phys. Rev. B 74, 144306 (2006).

[56] I. Quotane, E. H. E. Boudouti, and B. Djafari-Rouhani, Trapped-mode-induced Fano resonance and acoustical transparency in a one- dimensional solid-fluid phononic crystal, Phys. Rev. B 97, 024304 (2018).

[57] F. Nori and J. P. Rodriguez, Acoustic and electronic properties of one-dimensional quasicrystals, Phys. Rev. B 34, 2207 (1986).

[58] S. Tamura and J. P. Wolfe, Acoustic-phonon transmission in quasiperiodic superlattices, Phys. Rev. B 36, 3491(R) (1987).

[59] Y.-Y. Zhu, N.-B. Ming, and W.-H. Jiang, Ultrasonic spectrum in Fibonacci acoustic superlattices, Phys. Rev. B 40, 8536 (1989).

[60] I. Quotane, E. H. El Boudouti, B. Djafari-Rouhani, Y. El Hassouani, and V. R. Velasco, Bulk and surface acoustic waves in solid-fluid Fibonacci layered materials, Ultrasonics 61, 40 (2015).

[61] V. Laude, M. Wilm, S. Benchabane, and A. Khelif, Full band gap for surface acoustic waves in a piezoelectric phononic crystal, Phys. Rev. E 71, 036607 (2005).

[62] S. Benchabane, A. Khelif, J.-Y. Rauch, L. Robert, and V. Laude, Evidence for complete surface wave band gap in a piezoelectric phononic crystal, Phys. Rev. E 73, 065601(R) (2006).

[63] D. Yudistira, A. Boes, B. Djafari-Rouhani, Y. Pennec, L.-Y. Yeo, A. Mitchell, and J. R. Friend, Monolithic Phononic Crystals with a Surface Acoustic Band Gap from Surface PhononPolariton Coupling, Phys. Rev. Lett. 113, 215503 (2014).

[64] A. N. Darinskii and A. L. Shuvalov, Surface acoustic waves on one-dimensional phononic crystals of general anisotropy: Existence considerations, Phys. Rev. B 98, 024309 (2018).

[65] K. A. Ingebrigtsen and A. Tonning, Elastic surface waves in crystals, Phys. Rev. 184, 942 (1969). 
[66] S. V. Biryukov, Yu. V. Gulyaev, V. V. Krylov, and V. P. Plessky, Surface Acoustic Waves in Inhomogeneous Media (SpringerVerlag, Berlin, 1995).

[67] Y. B. Fu and A. Mielke, A new identity for the surfaceimpedance matrix and its application to the determination of surface-wave speeds, Proc. R. Soc. London, Ser. A 458, 2523 (2002).

[68] M. C. Pease III, Methods of Matrix Algebra (Academic, New York, 1965).

[69] P. Chadwick and G. D. Smith, Foundations of the theory of surface waves in anisotropic elastic materials, Adv. Appl. Mech. 17, 303 (1977).

[70] A. L. Shuvalov, E. Le Clezio, and G. Feuillard, The state-vector formalism and the Peano-series solution for modeling guided waves in functionally graded anisotropic piezoelectric plates, Int. J. Eng. Sci. 46, 929 (2008).
[71] A. L. Shuvalov and A. G. Every, Some properties of surface acoustic waves in anisotropic-coated solids, studied by the impedance method, Wave Motion 36, 257 (2002).

[72] V. I. Alshits, A. N. Darinskii, and J. Lothe, On the existence of surface waves in semi-infinite media with piezoelectric and piezomagnetic properties, Wave Motion 16, 265 (1992).

[73] V. I. Alshits, A. N. Darinskii, J. Lothe, and V. N. Lyubimov, Surface acoustic waves in piezocrystals: An example of surface wave existence with clamped boundary, Wave Motion 19, 113 (1994).

[74] http://www.ctscorp.com.

[75] X. Huo, S. Zhang, G. Liu, R. Zhang, J. Luo, R. Sahul, W. Cao, and Th. R. Shrout, Elastic, dielectric and piezoelectric characterization of single domain PIN-PMN-PT: Mn crystals, J. Appl. Phys. 112, 124113 (2012). 